\title{
Aeroacoustics research in Europe: The CEAS-ASC report on 2014 highlights
}

\section{Yves Detandt}

Free Field Technologies, Axis Park, rue E. Francqui 9, 1435 Mont-Saint-Guibert, Belgium

\section{A R T I C L E I N F O}

\section{Article history:}

Received 20 May 2015

Received in revised form

3 July 2015

Accepted 7 July 2015

Handling Editor: J. Astley

\begin{abstract}
A B S T R A C T
The Council of European Aerospace Societies (CEAS) Aeroacoustics Specialists Committee (ASC) supports and promotes the interests of the scientific and industrial aeroacoustics community on an European scale and European aeronautics activities internationally. Each year the committee highlights some of the research and development projects in Europe. This paper is the 2014 issue of this collection of Aeroacoustic Highlights, compiled from informations submitted to the CEAS-ASC. The contributions are classified in different topics; the first categories being related to specific aeroacoustic challenges (airframe noise, fan and jet noise, helicopter noise, aircraft interior noise) and two last sections are respectively devoted to recent improvements and emerging techniques and to general advances in aeroacoustics. For each section, the present paper focus on accomplished projects, providing the state of the art in each research category in 2014.

A number of research programmes involving aeroacoustics were funded by the European Commission. Some of the highlights from these programmes are summarised in this paper, as well as highlights funded by national programmes or by industry.
\end{abstract}

(c) 2015 Elsevier Ltd. All rights reserved.

\section{Introduction}

The CEAS-ASC is providing each year a report summarizing significant advances in aeroacoustic research fields. The present paper corresponds to the 2014 edition of this report. As for previous editions, it would be impossible to give a detailed view of the scientific activity in each research topics and the present paper focuses on novel advances and findings from accomplished projects funded by European, industrial or national programmes.

The paper is organised as a collection of contributions, divided into sections corresponding to the main topics of research in 2014.

In airframe noise (see Section 3), the research currently focuses on computational tools to validate the experimental benefits of new technologies applied for leading and trailing edges noise reduction, but also to model the jet-wing interactions. Section 4 reveals the research efforts in turbomachinery noise sources identification and on indirect combustion noise occurring in turbine stage.

In the field of helicopter noise, a contribution presents the research on optimizing and controlling the blade-vortex interaction noise. The elastic and compressibility effects on noise mapping are also investigated and presented in Section 5.

Three contributions are representing the advances in aircraft interior noise in Section 6. The current focus is on better understanding the boundary layer noise sources applied on the exterior part of the fuselage which may lead to

E-mail address: yves.detandt@fft.be 
improvements of semi-empirical models, but also on reducing the interior noise by means of efficient design of the panels or using active control systems.

The use of propellers for novel aircraft design is still considered as a viable option for the future. Section 7 presents novel techniques or fast methods which are appropriate for the optimization and the selection of relevant solutions at an early design stage.

Section 8 presents recent advances in experimental methods and numerical techniques. Section 9 is devoted to general concepts that would have an impact in different research areas.

Interested readers are invited to directly address their questions or comments to the authors who are mentioned at the end of each subsection.

\section{CEAS-ASC workshop}

The 18th CEAS-ASC workshop was held in Vilnius Gediminas Technical University, Lithuania on September 25-26, 2014, in conjunction with an International Acoustical Conference and X-Noise scientific activities. Organized by Professor Aleksandras Jagniatinskis, the workshop title was "Aircraft Noise Reduction by Flow Control and Active/Adaptive Techniques" and the scientific chairman was Professor Oleksander Zaporozhets (National Aviation University, Ukraine). The workshop had three keynote speakers:

- Victor Kopiev, TsAGI, Russian Federation on "On Instability Wave Control in Turbulent Jets",

- Vadim Tokarev, National Aviation University, Ukraine on "Experience of the National Aviation University in the Application of Active Noise Control in an Aircraft Cabin”, (presented by O. Zaporozhets)

- Dominique Collin, SNECMA, France on "Active Noise Technological Solutions in Aircraft Engine Design"

There were 8 contribution papers presented to 34 participants coming from 17 countries.

\section{Airframe noise}

\subsection{Mechanisms of trailing edge noise reduction by means of micro slots or porosity}

Trailing edge noise reduction by means of replacing the rear 11 percent of the chord c of an airfoil with porous material was investigated experimentally and numerically. As simple porosity streamwise oriented micro slots of 0.025 percent chord width were studied, periodic along the span at 0.125 percent chord spanwise spacing. The computation resolved this geometry. An inviscid nonlinear perturbation simulation about the RANS mean flow was done in which one single spanwise perturbation vortex was initiated in the upper boundary layer at about 85 percent chord. Fig. 1a depicts the perturbation pressure time history 45 percent chord below the trailing edge for the solid and the slotted edge. The solid edge shows a characteristic max-min pulse signature. The slotted edge shows two subsequent such signatures at reduced amplitude, because sound is generated at the leading edge of the slots and the airfoil's trailing edge. An impermeable horizontal splitter plane in the slots removes the effect of the slots. Fig. 2a shows the corresponding directivity. The reduction mechanism of the slots is inferred from the instantaneous pressure and velocity perturbation in Fig. 3. The vorticity of the vortex induces flow around the edge. For the solid edge the flow strictly points away from the edge in the lower part, representing sound while for the slotted edge, the flow is sucked through the slot back into the vortex system, see [13]. Fig. 2b shows the measured noise reduction for slots and various porous materials [36] in quantitative agreement with the computations. Apparently the mechanism is not driven, but only modified by viscosity.

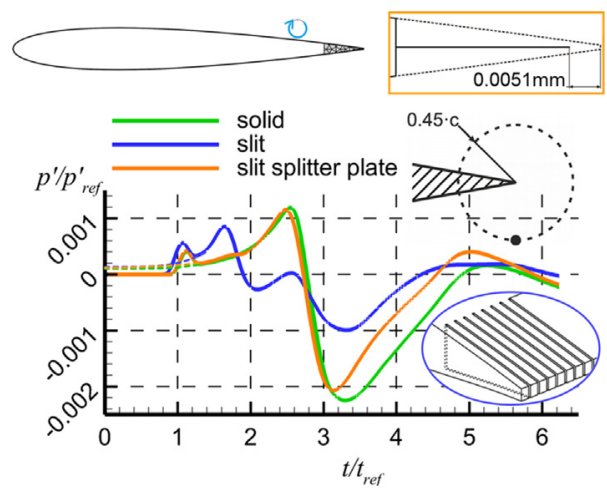

Fig. 1. Computed noise reduction of slotted trailing edge. Top: airfoil, right: splitter plane in slot, bottom: time pressure signature of vortex passing edge along upper boundary layer at $45 \%$ chord below trailing edge. 
(a)

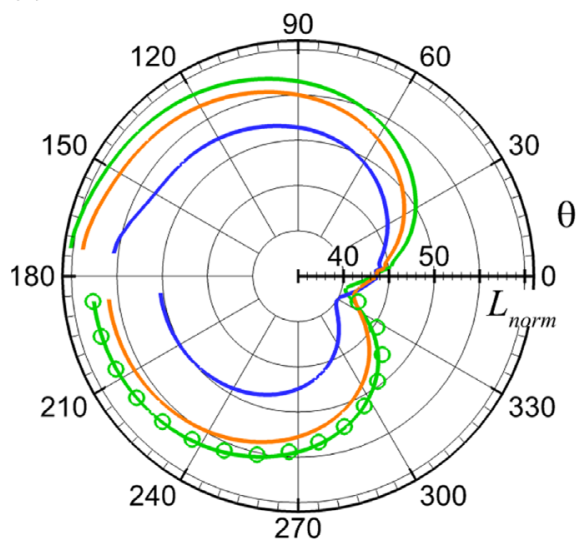

(b)

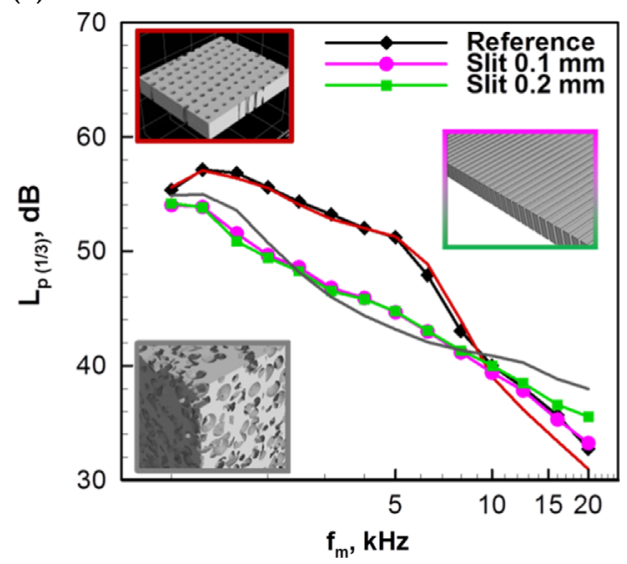

Fig. 2. Trailing edge noise: (a) computed directivity of trailing edge sound from spanwise vortex passage in upper boundary layer. Green line: solid edge, compared to analytical solution (symbols), blue: slotted, orange: slotted with split plane. (b) Third octave band spectra for trailing edge noise, based on elliptic mirror measurement in Acoustic Windtunnel Braunschweig, DLR. Pink/green: slotted edge, red: perforated sheet metal skin, grey: porous aluminum. (For interpretation of the references to color in this figure caption, the reader is referred to the web version of this paper.)

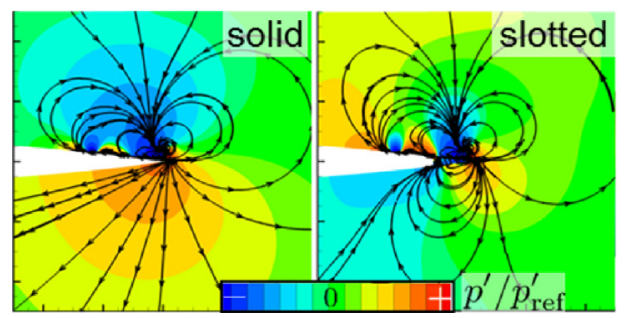

Fig. 3. Instantaneous pressure and velocity perturbation field at dimensionless time 2.18 (see Fig. 1). Left: solid edge displays acoustic particle velocity point away from edge, right: slotted edge, slot section, showing velocity reunifying with vortex system above edge; the driving vortex system above the edge is practically independent of the slots.

Written by Jan W. Delfs (jan.delfs@dlr.de), Benjamin Faßmann, Michaela Herr, DLR, Germany Michael Mößner, Institute of Fluid Mechanics, Technische Universität Braunschweig, Germany.

\subsection{Large-scale evaluation of low-noise slat technologies in OPENAIR}

Slat noise research activity within the EC co-financed project OPENAIR involved both experimental and numerical studies at the large (1:3.3-scaled) swept high-lift wing model F15LS [54,35]. Experiments were performed in the DNW-LLF (Large Low-speed Facility) to verify the noise reduction benefit of selected low-noise slat technologies under more realistic test conditions than in precursor projects. Moreover, the test data obtained served to extend numerical code validation to significantly higher Reynolds numbers. Comparisons of CAA (Computational Aeroacoustics) prediction results with the measurement data showed a generally good agreement, confirming applicability of the used codes [20,4] for low-noise design purposes (cf. Fig. 4).

Slat noise reduction concepts under review were (1) slat gap/overlap setting variations, and (2) an adaptive slat with the potential to reduce the gap width for noise reduction. Both technologies were proven highly efficient: sealing of the gap leads to a maximum 5-dB noise reduction at wing level, equivalent to a full elimination of the slat noise source (cf. Fig. 5). Optimized slat settings or an adaptive slat with partially closed gap are suited to reduce slat noise by about 2-3 dB at wing level while producing negligible aerodynamic impact at the operative test angles of attack within the linear polar region. When transposing these results to overall aircraft flight conditions, optimized slat settings or adaptive slats are expected to bring about a 0.5-0.6 EPNdB reduction of approach certification noise levels based on the conservative assumption that all other aircraft noise contributors than the slat are left untreated.

Written by M. Herr (michaela.herr@dlr.de), M. Pott-Pollenske, R. Ewert, D. Boenke, J. Siebert, J. Delfs, A. Rudenko, DLR, Germany, A. Büscher, H. Friedel, Airbus, Germany, I. Mariotti, Airbus, France.

\subsection{CAA computation of generic jet-flap interaction (JFI) configuration}

The objective of these CAA computations is the study of interaction between jet flow and flap or rather in general with an airfoil trailing edge and its effect on noise radiation. A generic JFI configuration has been designed for experimental and 

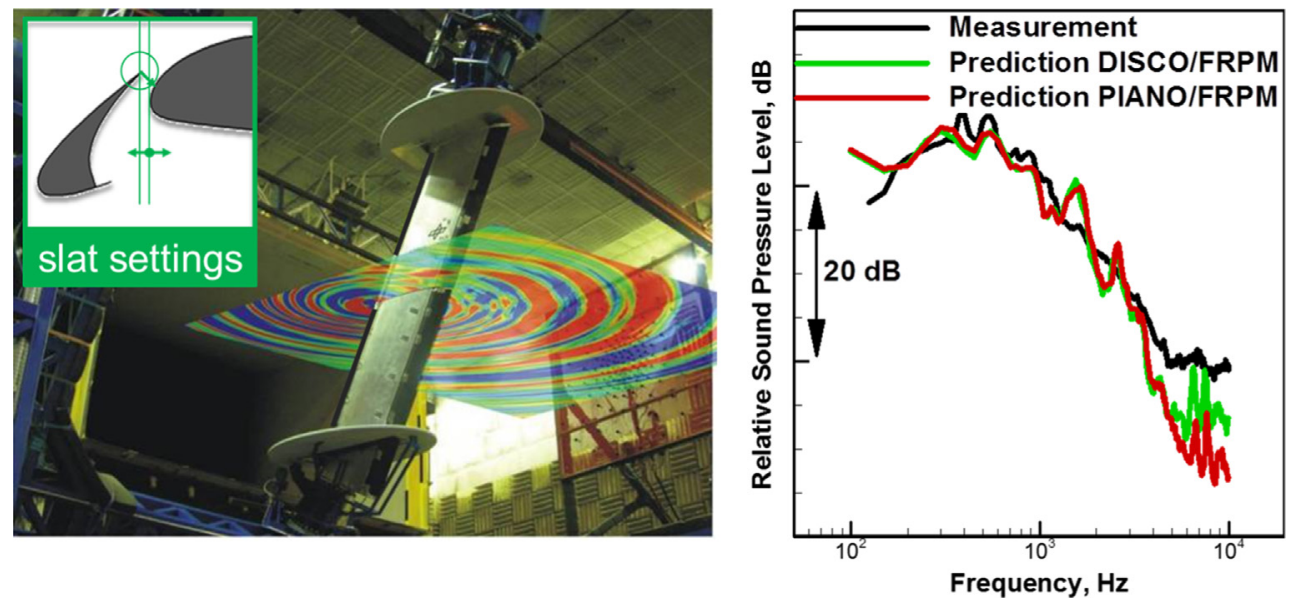

Fig. 4. F15LS installed in the DNW-LLF. Photographical composition with snapshot of a simulated 2D-slat noise sound field (left). Comparisons of measured and predicted slat noise narrowband spectra applying DLR's CAA codes with stochastic source models (right).

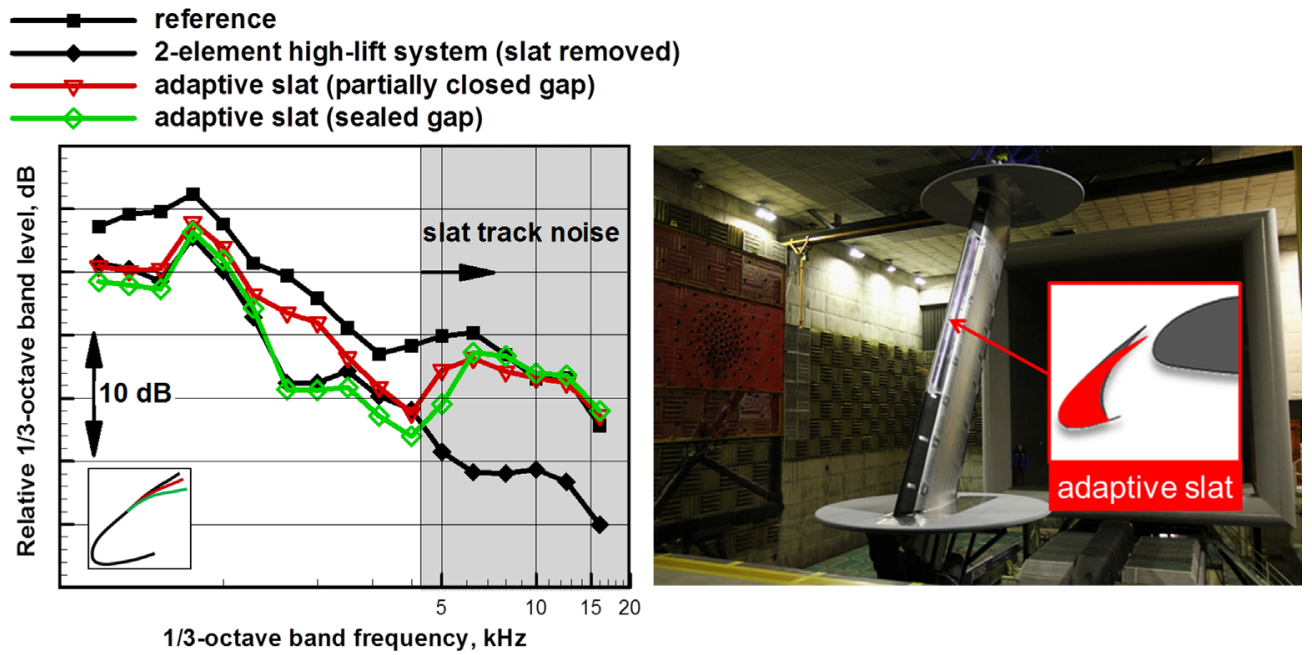

Fig. 5. Acoustic assessment of the adaptive slat technology at wing level; exemplary $1 / 3$-octave-band sound pressure spectra from array measurements at normal overflight observation angle.

numerical studies with the aim to generate a strong interaction as described in [55]. Such flow configurations are currently of high interest due to the latest trend in aircraft industries towards Ultra High Bypass Ratio (UHBR) engines. Because of a close installation of the engine below the airframe, the immersed trailing edge in the jet flow causes a significant radiation of jet induced trailing edge noise. These aeroacoustical constellations pose a relatively challenging task for noise prediction, since multiple noise-dependent parameters are composing a complex overall problem. The noise prediction of JFI requires the modelling of acoustical sources, which are able to reproduce three distinct noise generation mechanisms, i.e. the directly generated jet noise (fine- and large-scale noise) in the shear-layer and the airframe noise from the interaction of shear-layer vorticity with the trailing edge. In order to meet these requirements, the acoustical sources are modelled stochastically as vortical fluctuations on the basis of previously computed RANS solution with the means of Random Particle Mesh (RPM) method $[21,47]$. The representation of three different noise generation mechanisms, i.e. fine-scale noise, large-scale and flap noise is demonstrated in Figs. 6 and 7 with the FRPM/PIANO approach.

Written by A. Neifeld (andrej.neifeld@dlr.de), J.Dierke, R. Ewert, J.W. Delfs, DLR.

\section{Fan and jet noise}

\subsection{Experiments on axial compressors: time-resolved analysis of rotating instability modes}

Rotating instability (RI) occurs at off-design conditions in axial compressors, predominantly in rotor configurations with large tip clearances. Characteristic spectral signatures with side-by-side peaks below the blade passing frequency (BPF) are typically referred to RI located next to the clearance region (Fig. 8a, near-field) [37]. Each peak can be assigned to a dominant 


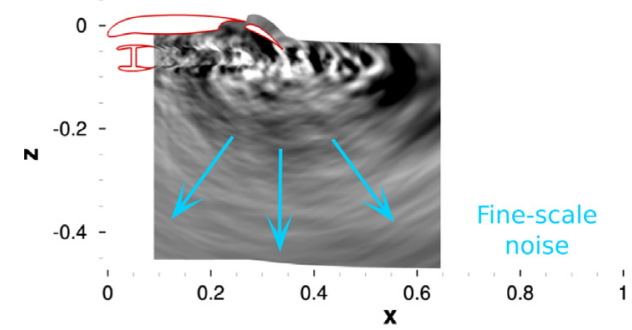

Fig. 6. Generic JFI configuration with a single stream jet $(\mathrm{Dj}=0.04 \mathrm{~m}, \mathrm{Maj}=0.8)$ below a two element airfoil in static condition computed with linearized Euler equations in combination with Tam and Auriault source model [62].

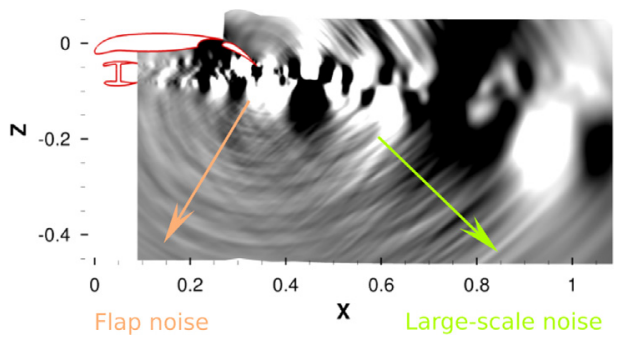

Fig. 7. Generic JFI configuration with a single stream jet $(\mathrm{Dj}=0.04 \mathrm{~m}, \mathrm{Maj}=0.8)$ below a two element airfoil in static condition computed with nonlinear Euler equations in combination with eddy relaxation source model [21].

(a)

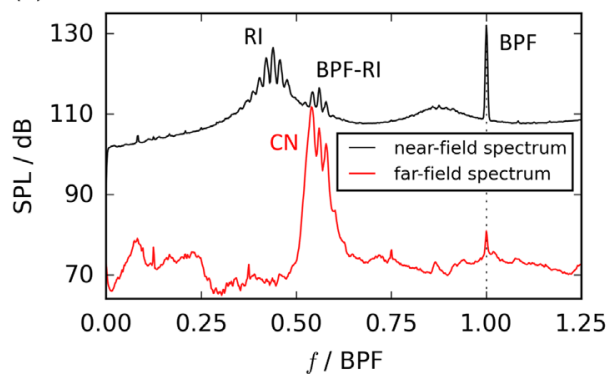

(b)

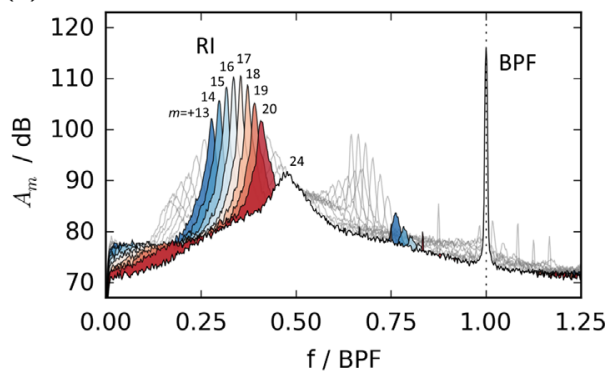

Fig. 8. Axial compressor: (a) measurements taken both in the near-field as well as in the far-field of the axial fan stage. (b) Circumferential mode amplitude distribution below the BPF measured in the near-field of the rotor with highlighted modes of order $m=+13, \ldots,+20$.

circumferential mode (Fig. 8b). RI is an indicator for critical operating conditions and potentially excites clearance noise (CN) caused by an interaction with the rotor (Fig. 8a, far-field) [37,50]. Earlier studies at an annular cascade pointed out that the RI modes of different circumferential orders occur stochastically distributed in time and independently from each other $[49,50]$. As a consequence, the validity of the existing RI models was questioned. An experimental study was conducted on a laboratory fan stage to verify these findings with regard to axial rotor configurations [50]. A mode decomposition using unsteady pressure measurements in sensor rings highlighted the spectral and modal RI patterns (Fig. 8b). A time-resolved analysis was applied to clarify the temporal characteristics of the dominant RI modes (Fig. 9a). It was proven by means of triggered results that the individual RI modes occur stochastically distributed in time without an interrelation between modes of different orders (Fig. 9b). This confirms the findings obtained on an annular cascade and corroborates the hypothesis already postulated by Pardowitz [49,51,50]: RI is associated to instability waves of different wavelengths. These are generated stochastically in a shear layer resulting from a back-flow extending over the whole circumference.

Written by Benjamin Pardowitz (Benjamin.Pardowitz@dlr.de), Ulf Tapken, Lars Enghardt German Aerospace Center (DLR) Institute of Propulsion Technology, Engine Acoustics Department, Germany.

\subsection{Numerical predictions of turbulence-cascade interaction noise using CAA with a stochastic model}

Turbulent flow interactions with the outlet guide vanes are known to mainly contribute to broadband noise emission of aeroengines at approach conditions. Onera has developed [11] a 3D CAA hybrid method for the simulation of the aeroacoustic response of an annular cascade impacted by a prescribed homogeneous isotropic turbulent flow. It is based on a time-domain Euler solver coupled to a synthetic turbulence model generating a suited inflow boundary condition. The 
(a)

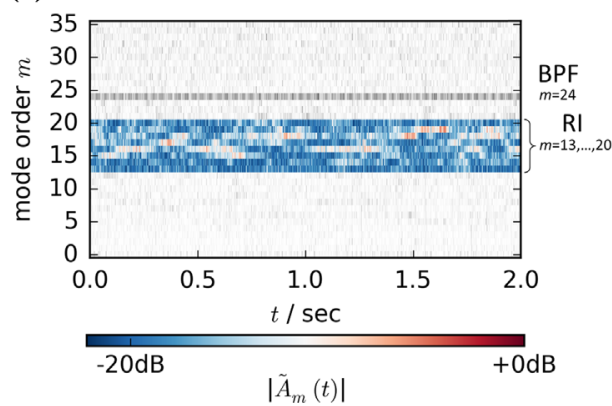

(b)

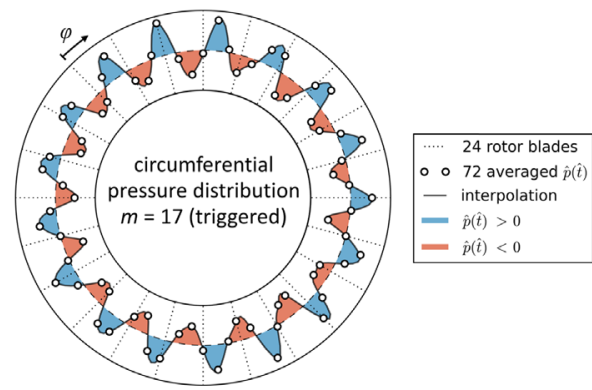

Fig. 9. Axial compressor: (a) time resolved circumferential mode amplitude distribution with highlighted modes of order $m=+13, \ldots,+20$. (b) Triggered results by using the dominant RI mode of order $m=17$.

(a)

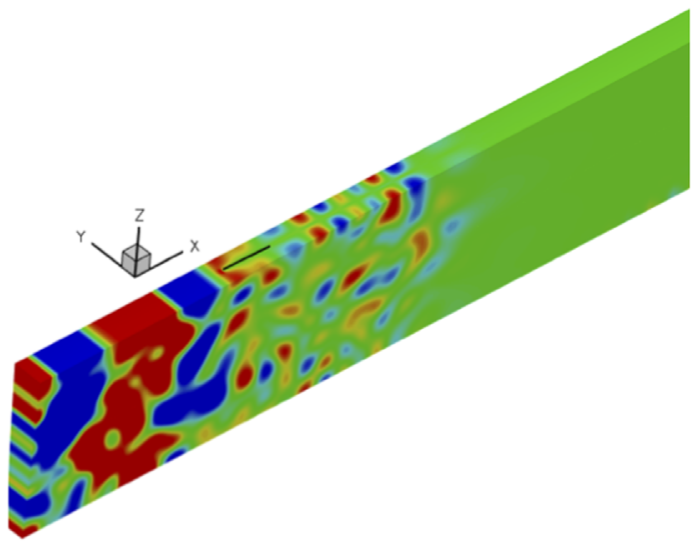

(b)

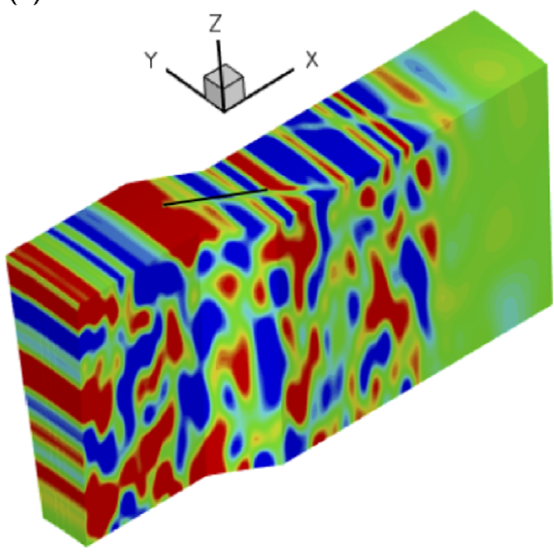

Fig. 10. (a) Snapshot of azimuthal velocity disturbances provided by 3D CAA computation (single vane channel) of ECL turbulence-cascade case. (b) Snapshot of azimuthal velocity disturbances provided by 3D CAA computation (single vane channel) of Atassi turbulence-cascade case.

fluctuating pressure over the airfoil surface provided by CAA is used as an input to a Ffowcs-Williams and Hawkings integral method generalized to annular duct propagation (Goldstein's formulation) to calculate the radiated sound field. Euler computations were first validated against an academic CAA benchmark in the case of an harmonic gust interacting with an annular flat plate cascade. Then, simulations have been applied to turbulence-cascade interactions for annular configurations, in uniform related to a laboratory experiment performed by Ecole Centrale de Lyon [53] (Fig. 10a) and a swirling mean flow related to a benchmark proposed by Atassi [3] (Fig. 10b). Numerical results in terms of sound power spectra in the outlet duct have been compared to semi-analytical and numerical solutions, and to available measurements. A fairly good agreement has been obtained for the two cases (Fig. 11a and b). Next step will be to apply this methodology to the Nasa SDT fan-OGV configuration as planned for the 2nd Workshop related to turbofan broadband noise prediction [19].

Written by C. Polacsek (cyril.polacsek@onera.fr), Onera, France, V. Clair, ISVR, United-Kingdom, T. Le Garrec, G. Reboul, Onera, France, M. Jacob, ECL, France.

\subsection{On the generation and propagation of multiple pure tones (MPT) inside turbofans at transonic regime}

Whereas the sound radiated from the inlet of turbofans is mainly due to rotor-stator interactions in approach flight, the shock waves (or N-waves) emitted by the rotor at transonic rotation speeds can be a dominant noise source during takeoff and climb. Two key aspects must be considered to describe the shock-wave propagation in the inlet of a transonic turbofan. The first issue is the N-wave propagation, the second is the MPT generation. Onera studied the both aspects. Concerning the $\mathrm{N}$-wave propagation, Onera [63] compared the most advanced analytical methods in the time and frequency domains for two representative turbofan configurations (named FANPAC and MASCOT2). The reliability of each method has been discussed through a comprehensive analysis and validated using experimental data. Concerning the MPT generation, a statistical MPT [52] generation method has been successfully implemented and tested on the FACPAC turbofan model. The McAlpine and Fisher MPT [43] generation method has been investigated and tested on the full scale MASCOT2 engine from Snecma. During the test rig, not only the temporal signals were recorded at two different planes, but also the individual blade stagger angles. These data were obtained at several rotational speeds. From this test campaign, Onera developed an 
(a)

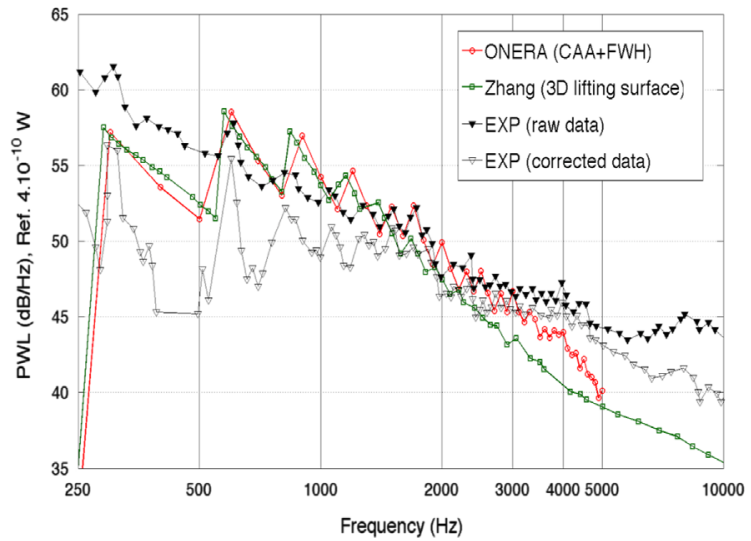

(b)

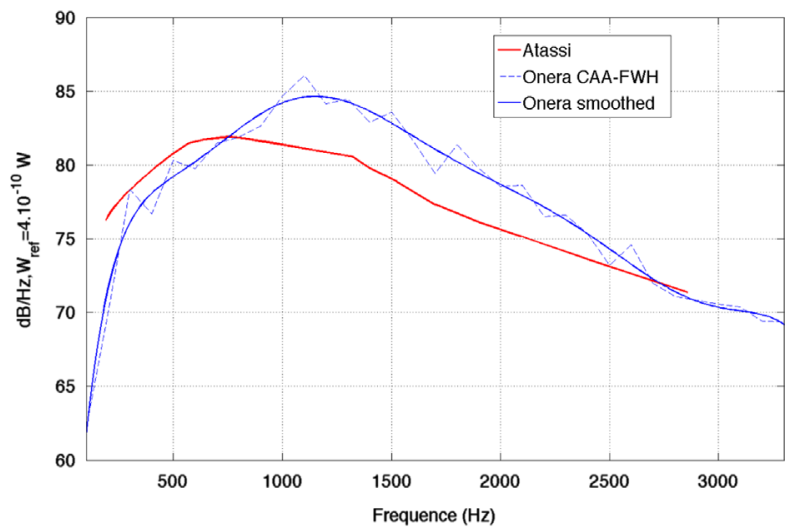

Fig. 11. (a) Outlet duct PWL spectra $(\mathrm{dB} / \mathrm{Hz})$ provided CAA+FWH (Onera), 3D lifting surface method (Zhang), and compared to experiments (raw and corrected measurements). (b) PWL spectra $(\mathrm{dB} / \mathrm{Hz}$ ) provided by $\mathrm{CAA}+\mathrm{FWH}$ (raw and smoothed Onera solutions) and compared to Atassi reference solution.
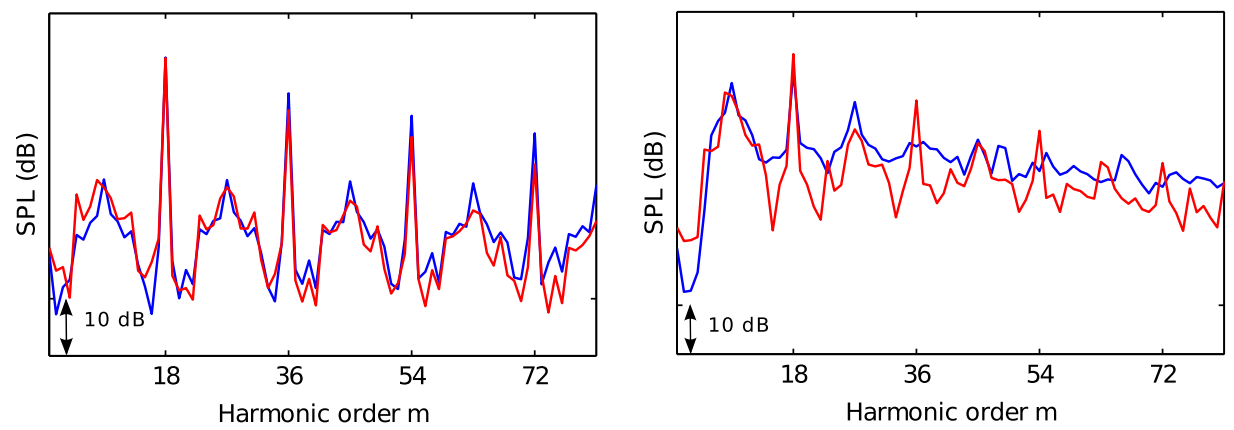

Fig. 12. Comparison between measured spectra (red) and computed spectra deduced from the stagger angles (blue) for wall-mounted microphones near the fan (left) and near the intake (right). (For interpretation of the references to color in this figure caption, the reader is referred to the web version of this paper.)

original approach of MPT generation based on real blade stagger angles. This MTP generation method has been successfully applied to the MASCOT2 engine model (see Fig. 12), and allows to predict in a simple way the spectrum shapes near the fan and at the intake for different blade arrangements around the wheel.

Written by Johan Thisse (johan.thisse@onera.fr), Cyril Polacsek, Onera, France, Serge Léwy, Onera, France, Anthony Lafitte, Snecma, France.

\subsection{Modelling combustion noise in gas turbines}

Flame unsteadiness in the combustors of gas turbines generates noise via two mechanisms. The first is direct noise, whereby acoustic waves are generated directly. The second is indirect noise, often known as "entropy noise", whereby temperature fluctuations are generated, advect downstream and upon rapid acceleration at the turbine inlet generate new acoustic waves. For both mechanisms, some noise eventually exits the turbine, contributing to an increasingly important component of aero-engine noise, while some propagates upstream, causing further flame perturbations and potentially thermoacoustic instability. We have developed models for three phenomena relevant to combustion noise:

- The advection of entropy waves from the flame to turbine inlet (see Fig. 13a). We have shown that advection by a fully developed turbulent flow results in shear dispersion, giving a Gaussian-shaped impulse response that can be inferred from the mean velocity profile. For typical gas turbine combustors, this implies that strong entropy waves remain at turbine inlet [45].

- The generation of indirect noise by entropy wave acceleration (see Fig. 13b). Using asymptotic approximations, low frequency models for transmitted direct and indirect noise through a nozzle have been derived [25]. Very recently, we have developed the first models for reflected and transmitted noise through a thin annular nozzle supporting plane and circumferential waves, which are valid for any nozzle geometry and frequency [17].

- The effect of entropy noise on thermoacoustic instability (see Fig. 14). We have shown that entropy noise can affect combustor thermoacoustic modes via mode switching, mode destabilisation/stabilisation and a purely acoustic-entropic instability [26]. 
(a)

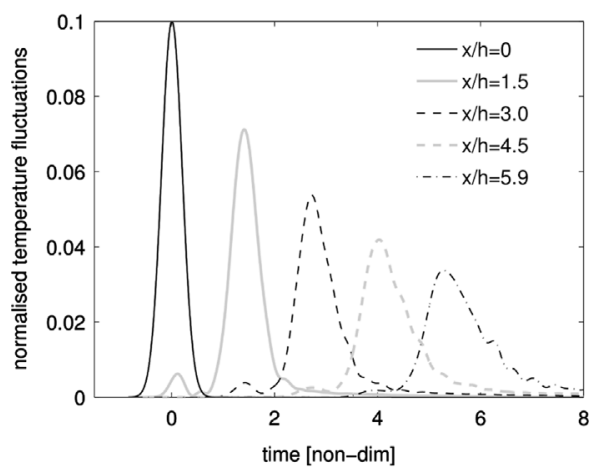

(b)

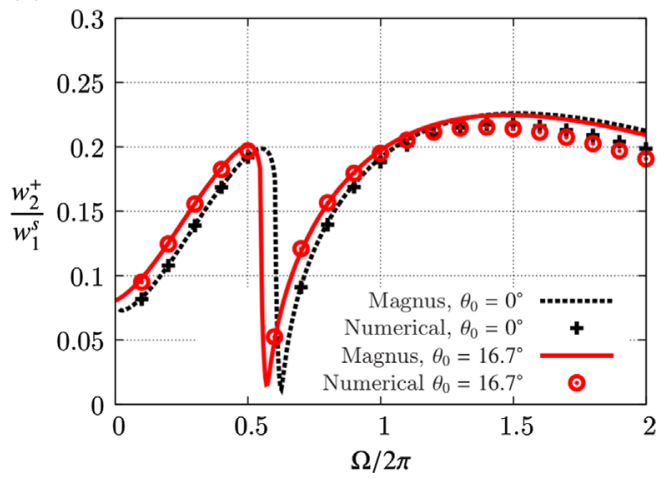

Fig. 13. (a) The approximate Gaussian impulse response of temperature fluctuations, shortening and spreading as they advect downstream in a channel flow. $x / h$ denotes distance downstream of the temperature impulse. (b) The magnitude of the entropy wave to transmitted acoustic wave transfer function, for an entropy wave incident upon a choked annular nozzle. Circumferential mode $n=4$. Magnus denotes modelling prediction, numerical denotes LEE simulation prediction.
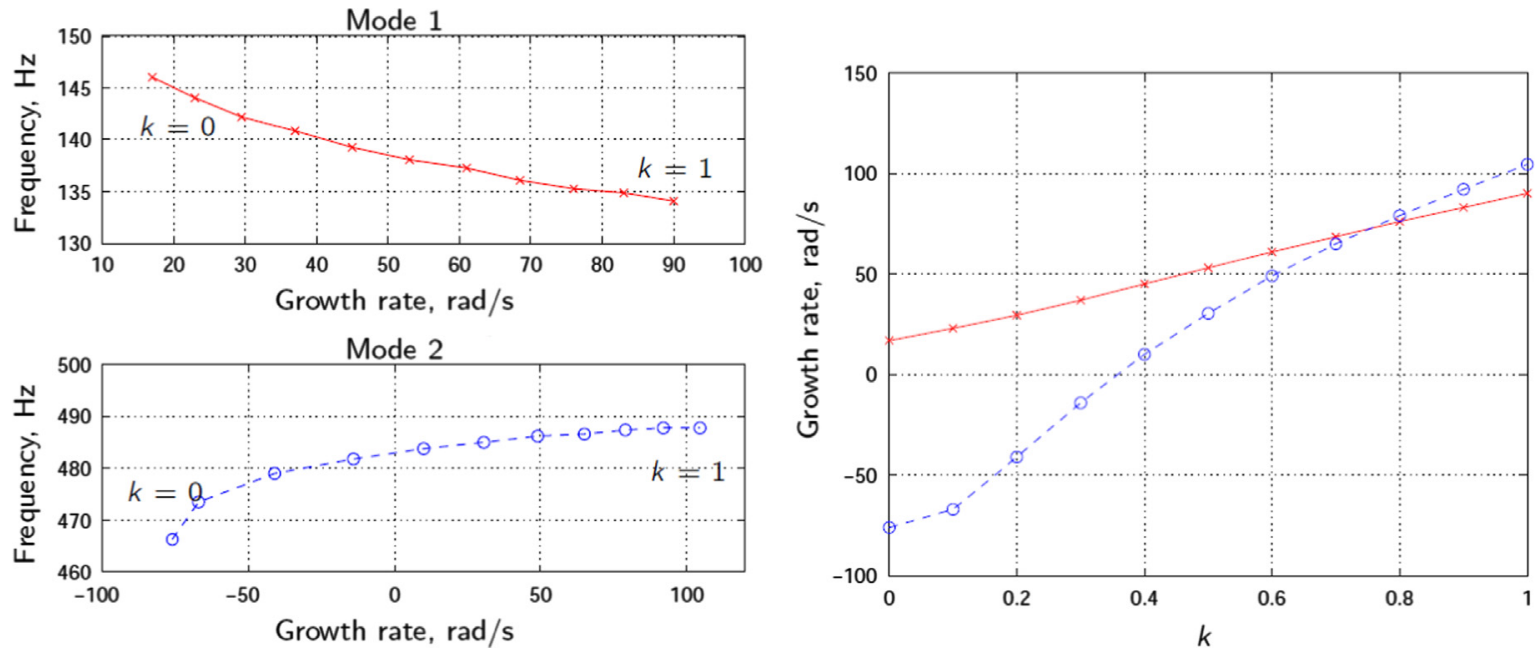

Fig. 14. The effect of reflected entropy noise on the first two thermoacoustic modes of a model combustor. k denotes proportion of entropy wave strength remaining at exit. Mode destabilisation and mode-switching are evident.

Written by Aimee S. Morgans (a.morgans@imperial.ac.uk), Chee Su Goh, Ignacio Duran, Jeremy A. Dahan Department of Aeronautics, Imperial College London, United Kingdom.

\section{Helicopter noise}

\subsection{Parallel blade-vortex interaction modelling for helicopter rotor noise control synthesis}

A methodology for alleviation of loads and corresponding emitted noise generated by blade-vortex interaction (BVI) phenomena occurring on helicopter main rotors has been presented in [6]. A multi-cyclic, optimal control approach is applied to drive high-frequency harmonic blade pitch actuation aimed at generating unsteady aerodynamic loads able to suppress/alleviate those caused by BVI. The numerical identification of the blade pitch optimal control law is obtained through a novel efficient process that relies on numerical simulations provided by rotor aerodynamics/aeroacoustics computational tools extensively validated in the past [6,23]. It is based on two-dimensional, equivalent modelling of BVI phenomena, and yields optimal high-frequency harmonic blade pitch actuation as a function of high-frequency harmonic loads measured at a characteristic blade section [44]. The results shown in [6] demonstrate that the presented control approach is a viable mean to reduce BVI noise (see, for instance, Fig. 15).

Written by Sara Modini (saramodini@gmail.com), Giorgio Graziani, Giovanni Bernardini, Massimo Gennaretti; Roma Tre University, Roma, Italy. 
(a)

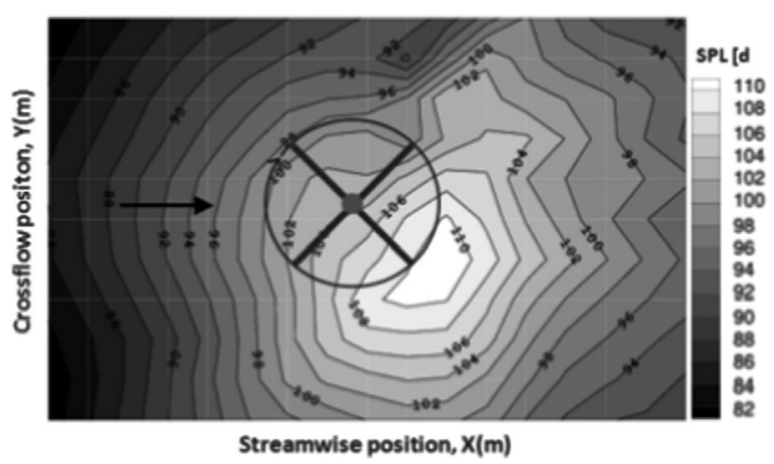

(b)

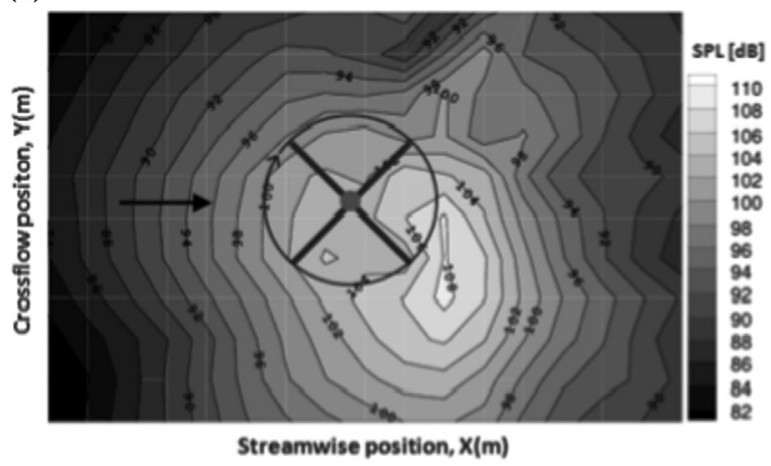

Fig. 15. Contour plot of BVISPL (sound pressure level associated to 7-40 blade passage frequencies) generated by controlled and uncontrolled rotor on a plane located $1.25 \mathrm{R}$ below the rotor disc.

\subsection{Rotor noise radiation under influence of blade deformation, compressibility and different fuselage modelling}

The influence of the elastic rotor blade deformation and the aerodynamic interference from the fuselage on the rotor aerodynamics as well as rotor noise characteristics was studied in [68]. A B0105 Main Rotor/Tail Rotor/Fuselage (FUS) configuration is chosen for the numerical simulations. A coupling of unsteady free wake 3-D panel method (UPM) [67] and Airbus Helicopters' (formerly: Eurocopter) rotor code HOST was conducted to account for the effect of elastic blade deformation as well as compressibility correction. The effect of fuselage is simulated by using two fuselage models in UPM, (1) potential theory in form of a panelized fuselage and (2) an analytic fuselage influence formulation derived from isolated fuselage simulation based on (1). The advantage of (2) is in its computational efficiency. Fig. 16a shows high frequency components of blade vortex interactions (BVI) are captured well by incorporating blade deformation (HOST + UPM) and Simplified compressibility correction (Prandtl-Glauert) shows relatively good agreement with CFD (for example at $90^{\circ}$ azimuth angle). The aerodynamic improvements are also reflected in BVI noise contours shown in Fig. 16b. Fig. 17a shows Increasing the complexity of configuration improves simulation results and introducing fuselage improves maximum noise level as the improvement of loading noise as indicated in Fig. 17b.

Written by Yin, Jianping (jianping.yin@dlr.de), van der Wall, Berend G., Wilke, Gunther A. DLR, Germany.

\section{Aircraft interior noise}

\subsection{Numerical and experimental investigations on the acoustic power radiated by aluminium foam sandwich panels}

Aluminium Foam Sandwich (AFS) panels are a new class of flat structures, constituted by two aluminium layers with a core made by foamed aluminium. Their excellent combination of properties, arising from the metallic nature of the matrix and from the porosity behaviour of the foam core, guarantees high specific characteristics. In this paper experimental and numerical estimation of the radiated acoustic power was carried out on two configurations of AFS panels having same in plane dimensions but different total thickness dimensions and percentage of porosity in the foam [42,5,66]. The experimental test was performed through a sound intensity technique in the range $0-1000 \mathrm{~Hz}$, summing the power radiated by each elementary cell in which the panel was subdivided. Test results showed a good matching between the natural frequencies, obtained from previous modal analysis tests [16], and the peaks of the maximum amplitude of the measured acoustic power. Furthermore, the experimental phonometric maps were also compared to the structural modal shapes, highlighting the possibility of determining the dynamic characteristics of a structure through acoustic tests. The experimental results were also used to validate the numerical finite element model, in which the radiated acoustic power was calculated through the discrete formulation of Rayleigh integral for flat radiator. The numerical-experimental correlation was quite good, showing the capability of the model to exhibit the same trend of the experimental results (Fig. 18).

Written by Petrone Giuseppe (giuseppe.petrone@unina.it), D' Alessandro Vincenzo, Franco Francesco, De Rosa Sergio, University of Naples Federico II (Italy).

\subsection{Tiltrotor cabin noise control through smart actuators}

Abatement of tonal noise inside the cabin of a mid-range tiltrotor aircraft, as generated by the propulsive system, is examined in [7]. The problem is multidisciplinary, involving interactions among exterior noise field, fuselage elastic dynamics, interior acoustics and control system. A stiffened fuselage, with piezoelectric patches embedded into the structure, is supposed to be impinged by the aeroacoustic field generated by propellers and forced by the wing/pylon/ proprotor vibratory loads at the wing-fuselage attachment. An optimal LQR cyclic control formulation, coupled with a 
(a)
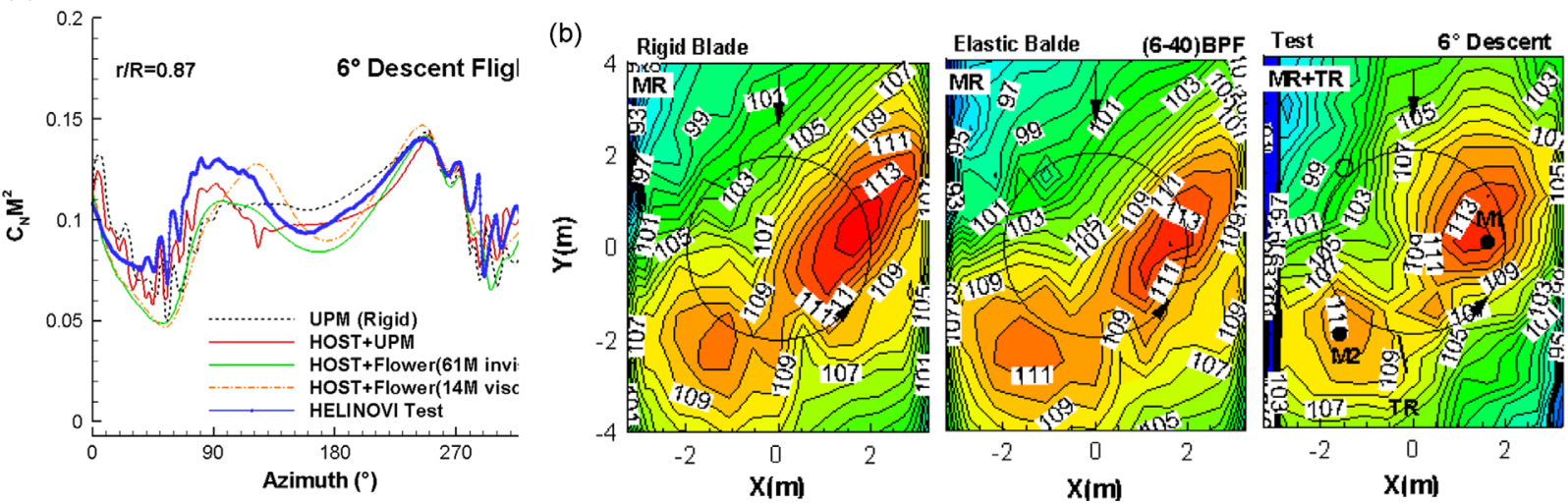

Fig. 16. (a) MR Normal force coefficient at $0.87 \mathrm{R}$ for $6^{\circ}$ descent flight. (b) Simulated and measured (right) BVI noise contours

(a)

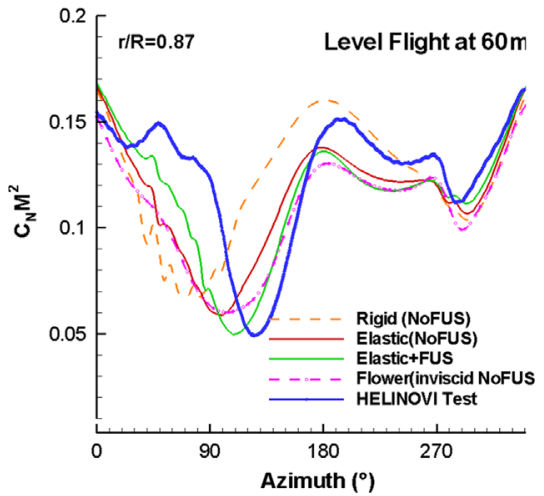

(b)

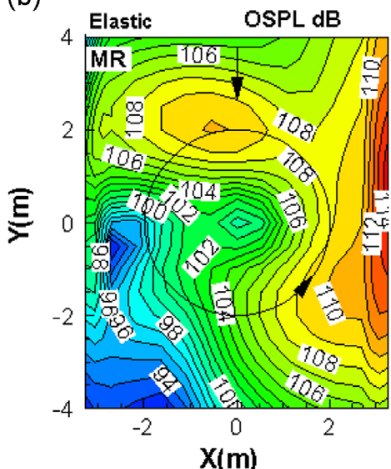

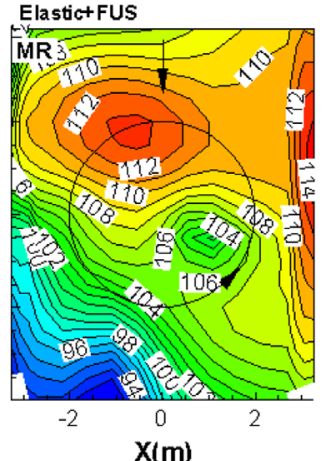

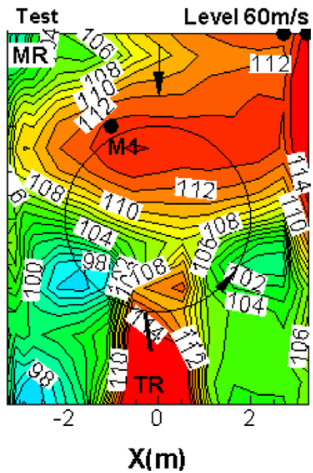

Fig. 17. (a) Normal force coefficient at $0.87 \mathrm{R}$ for $60 \mathrm{~m} / \mathrm{s}$ level flight. (b) Simulated and measured (right) OSPL noise contours for various fuselage modeling.

(a)

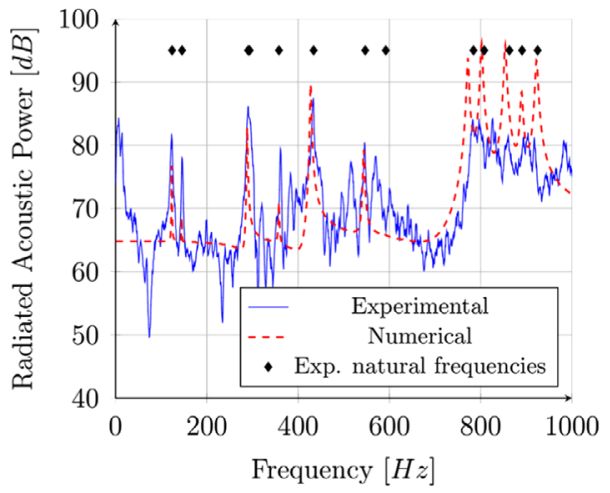

(b)

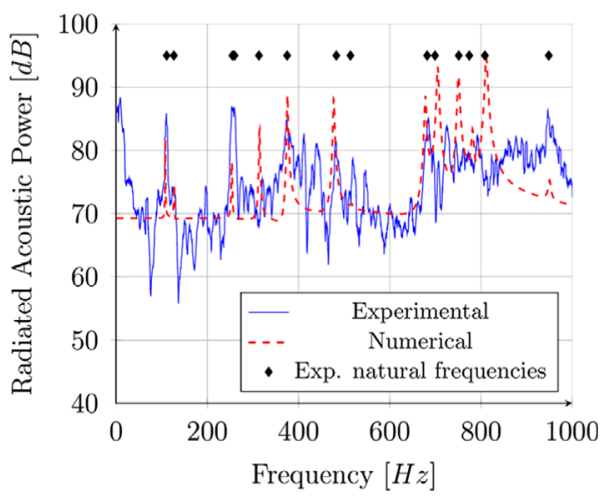

Fig. 18. Radiated acoustic power vs. frequency for two different panels.

genetic optimization algorithm and a comprehensive aeroacoustoelastic model, is applied to synthesize a control law driving the smart actuators so as to alleviate cabin noise. The aeroacoustoelastic model is obtained by combining cabin interior acoustics, fuselage smart shell dynamics and wing/pylon/proprotor aeroelasticity described through modal approaches, with the exterior pressure field provided by boundary element method solvers [22,23]. Numerical simulations show that the proposed control strategy yields satisfactory cabin noise alleviation and, at the same time, proves its robustness with respect to uncertainties/variations in the stiffness and mass parameters characterizing the system (Fig. 19).

Written by Giovanni Bernardini (g.bernardini@uniroma3.it), Claudio Testa, Massimo Gennaretti, University Roma Tre, Roma, Italy. 


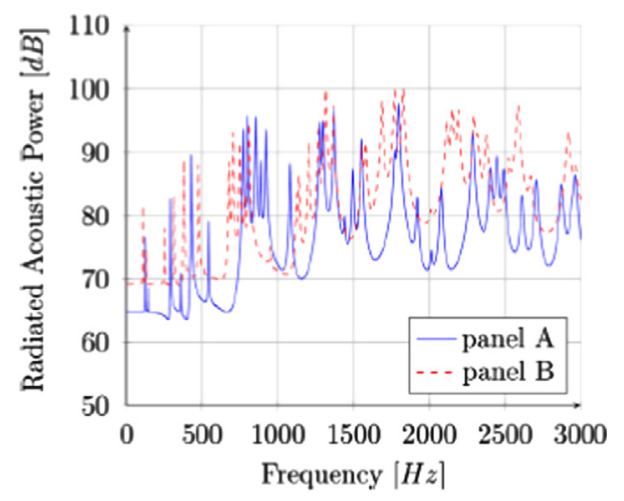

Fig. 19. Numerical radiated acoustic power vs. frequency of the panels A and B.

\subsection{Listening to turbulence: measuring coherence decay at different positions on an aircraft in cruise flight}

Measurements of the coherence length were conducted using a pressure transducer array at three different positions on an Airbus Model A320 aircraft [31]. The 30-transducer-array was installed in three consecutive window banks that were located in the front-, mid- and aft section of the fuselage. Coherence was obtained from the pressure data by using Welchs method and then fitting a two-dimensional negative-exponential surface through the measured coherence at all variations of transducer separation. The measured coherence length was normalized with the estimated boundary layer thickness and displayed over Strouhal number. In the front- and aft-section, the resulting normalized characteristic was alike, giving a maximum coherence length of approximately six times the boundary layer thickness. In the mid-section, the maximum coherence length was considerably lower, which was believed to occur due to an overestimation of the boundary layer thickness in the region of accelerated flow in close proximity to the wing. An evaluation of the convective velocity in the wavenumber domain showed no difference between the three positions throughout the Strouhal number region under consideration. The measured coherence lengths were compared to values predicted by the model of Efimtsov [18]. The predicted values were considerably lower than the measured values. This is astonishing since the coherence length values from this analysis might still underestimate the true values [48]. Besides different flow conditions, the difficulty of taking the flow direction into account and the large potential of error when not doing so are considered as an explanation for the difference (Figs. 20 and 21).

Written by Stefan Haxter (stefan.haxter@dlr.de), Carsten Spehr, DLR, Germany.

\section{Propeller noise}

\subsection{Installation effects of a propeller mounted on a wing with Coanda flap}

Within the framework of the regionally funded German project "Brgernahes Flugzeug (Metropolitan Aircraft)", the effect of installation on propeller noise was investigated. The chosen configuration consists of a nine bladed propeller in tractor configuration with a high-lift wing. Tests in the new acoustic wind tunnel DNW-NWB provided a valuable dataset for validation purpose of numerical codes. The dataset consists of parameter variations of angle of attack, wind tunnel velocity, rotational speed, pitch angle. Details of the measurements can be found in [1]. The CAA computations were based on a new highly efficient concept to represent the propeller as a perturbation consisting of rotating mass and momentum source distributions fed by CFD RANS data on the propeller blades, thus avoiding rotating grids. This approach will allow quantifying the installation effect at propeller driven aircraft especially to assess design changes with moderate computational effort. Details of the CAA implementation and computations can be found in [14]. Around 9 propeller revolutions are computed on 168 CPUs in less than 2 days, which is reasonably fast to enable parameter variations in a design process. The acoustic wind tunnel tests and respective CAA computations were consistent, Fig. 23a and b. Also the validation showed good agreement (Fig. 22b). As a result significant source noise installation effects were found in the mentioned generic installed propeller configuration. The particular tractor propeller/ high-lift wing arrangement showed excess noise due to the impingement of the propeller tip vortices on the (swept) wing nose and trailing edge flap.

Written by J. Dierke (juergen.dierke@dlr.de) DLR, Germany R.A.D. Akkermans, ISM, TU Braunschweig, Germany J.W.Delfs, DLR, Germany.

\subsection{Experimental and numerical techniques for rotating machinery noise prediction and optimization}

Amongst the aeroacoustic activities that were ongoing at the von Karman Institute for Fluid Dynamics (VKI), three topics have been the subject of more intensive developments in 2014: the experimental modal identification of ducted rotor noise, 
(a)

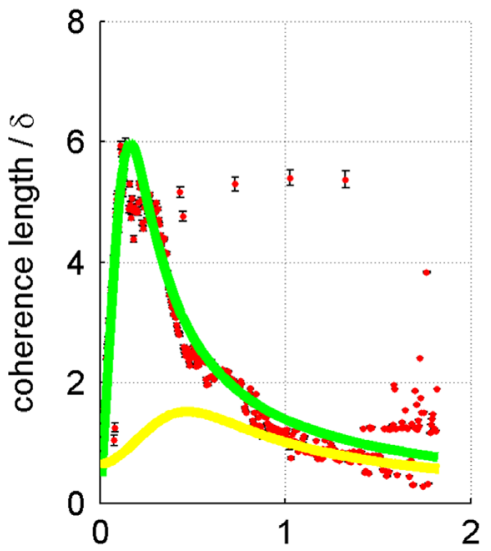

Strouhal number, $\omega \delta^{*} / \mathrm{u}_{\infty}$ (b)

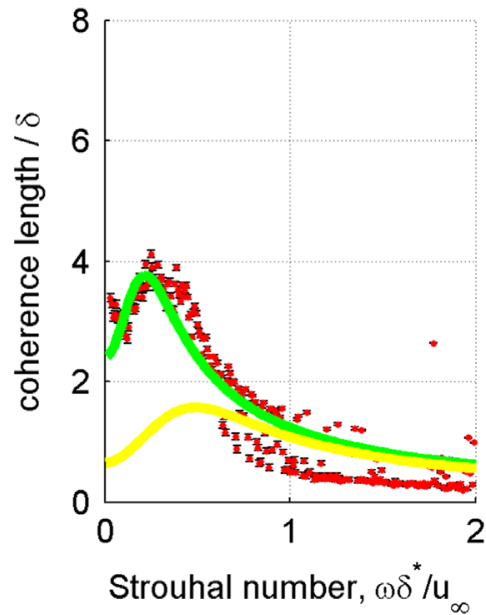

(c)

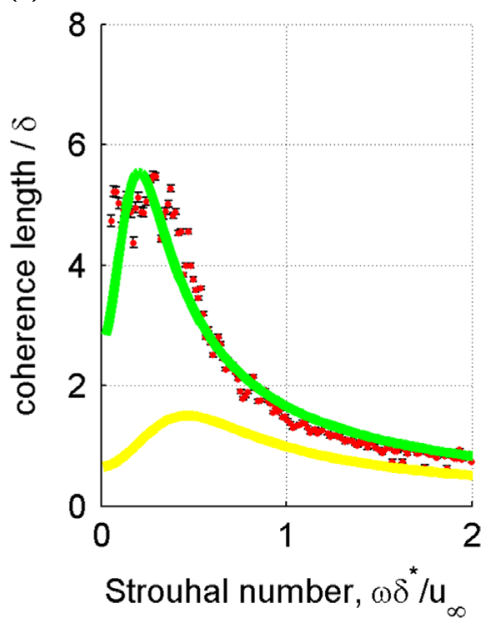

Fig. 20. Normalized coherence lengths from measurement (red dots), predicted values from the Efimtsov model (yellow), and adapted model (green) for the front- (a), mid- (b), and aft-section (c). (For interpretation of the references to color in this figure caption, the reader is referred to the web version of this paper.)

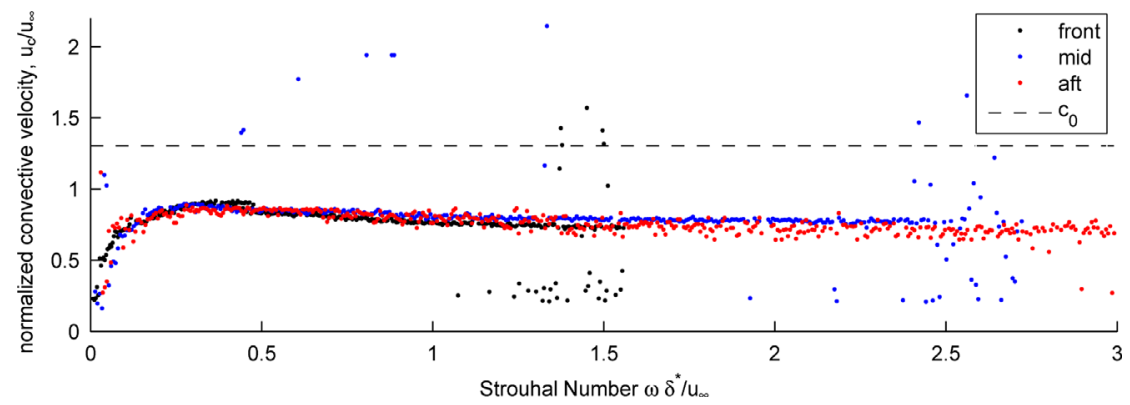

Fig. 21. Convective velocity normalized to $u_{\infty}$. The horizontal black line shows the speed of sound normalized to $u_{\infty}$.

(a)

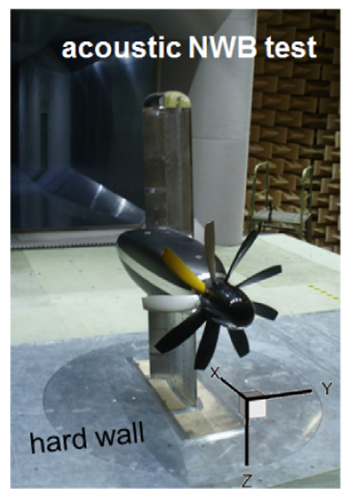

(b)

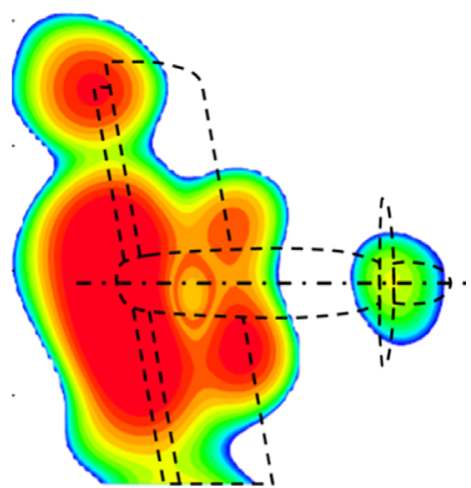

Fig. 22. Experimental measurements. (a) Wind tunnel setup with nine-bladed propeller, High-lift wing with Coanda flap and ground plate. (b) Sound radiation from installed propeller, measured array source noise.

the modelling of wake-blade interaction noise in ducted contra-rotating rotors, and the numerical extension of analytical modelling approaches for gust-airfoil interaction noise.

Modal identification techniques are being developed in order to decompose the noise emitted by an Environmental Control System blower into duct modes. This was achieved including not only the plane wave mode, but also the first two azimuthal and the first radial modes [61], in collaboration with KTH.

Still related to rotating machinery noise, a fast-running model was implemented for the optimization by evolutionary algorithms of tonal noise due to wake interaction in contra-rotating rotors. A synthetic wake generation algorithm has been implemented using a semi-empirical wake model (Fig. 24a). RANS calculations were also performed, requiring an 
(a)

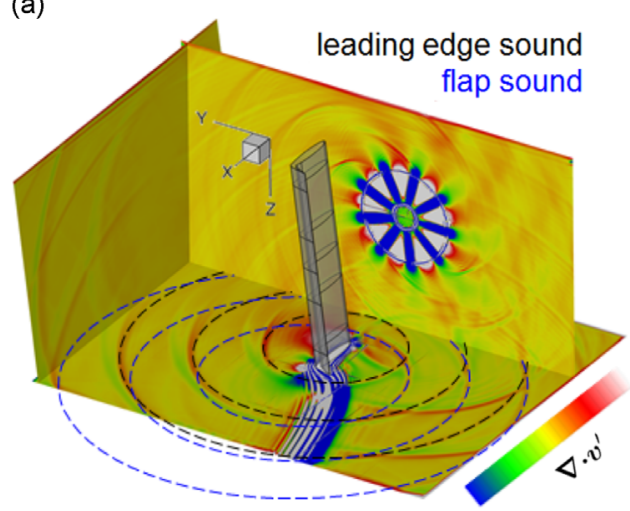

(b)

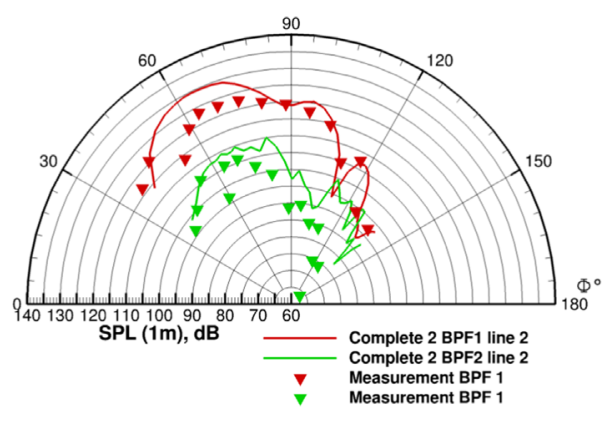

Fig. 23. (a) Sound radiation from installed propeller: CAA simulation (dilatation), blue/black: wave system originating from flap/wing nose. (b) Directivity comparison of measurement data with computed data $\left(V_{\mathrm{inf}}=51 \mathrm{~m} / \mathrm{s}, \beta=30^{\circ}, \alpha=-5^{\circ}, N_{p}=71,441 / \mathrm{min}\right)$. (For interpretation of the references to color in this figure caption, the reader is referred to the web version of this paper.)
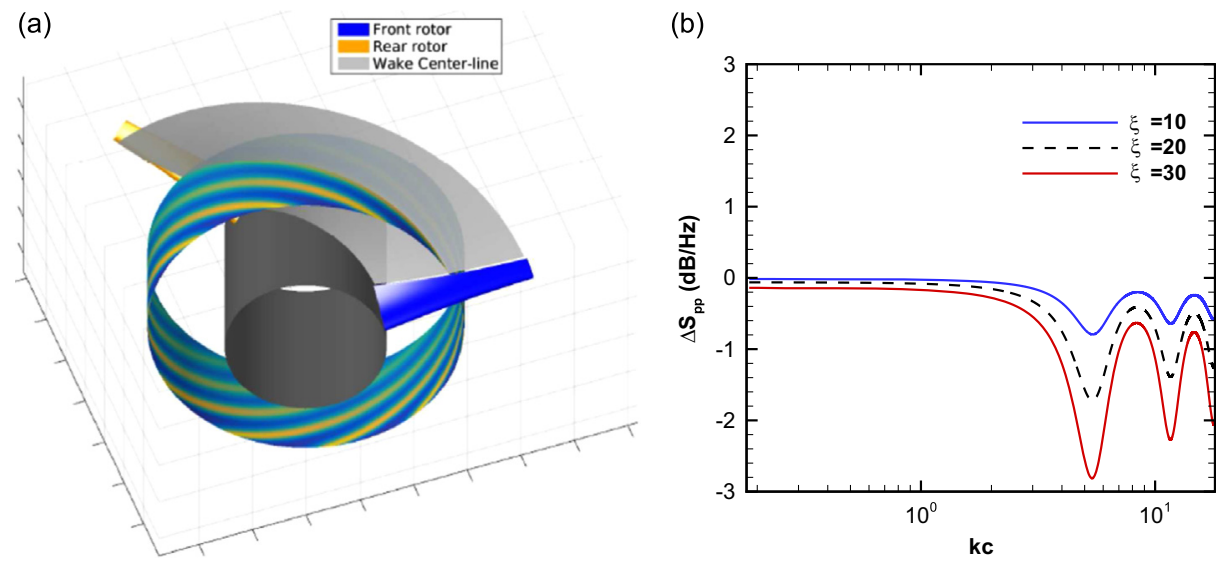

Fig. 24. (a) Synthetic wake generated for the VKI contra-rotating fan. Visualization of the wake center-line (grey) and of the velocity disturbance at midspan. (b) Difference between the gust-interaction noise spectra for a cambered airfoil and its linearized flat plate approximation. Camber angle $=10^{\circ}$ (continuous blue), $20^{\circ}$ (dashed black) and $30^{\circ}$ (continuous red). (For interpretation of the references to color in this figure caption, the reader is referred to the web version of this paper.)

extrapolation of the azimuthal Fourier decomposition of the wake profiles from the mixing plane to the downstream rotor [28].

This gust-airfoil interaction noise problem, usually treated using linearized airfoil theories, has been solved using an innovative iterative boundary element procedure, similar to the Schwartzchild approach [60], to account for more realistic geometrical effects, in the compressible regime and including the contribution of oblique gusts. The results of these predictions are qualitatively in agreement with the literature, showing however noticeable differences for high Helmholtz numbers [12] (see Fig. 24b).

Written by Christophe Schram (christophe.schram@vki.ac.be), Leandro De Santana, Gabriele Grasso, Joao Aguiar, von Karman Institute for Fluid Dynamics, Belgium.

\section{Techniques and methods in aeroacoustics}

\subsection{About the ambiguity of causality correlation results for noise source localization in cold jets}

Interpreting the coefficient matrix resulting from the correlation between turbulent flow quantities obtained in jet-flows and far-field pressure fluctuations can be ambiguous in regions with coherent structures dominating the flow-topology $[8,34]$. The aim of the present study is to contribute to the understanding of results from this so-called causality correlation approach. The sound radiated into the far-field is calculated using a free-space Green's function for correlated monopoles convecting with a constant speed along a jet axes. The amplitude of the modeled source is damped by an envelope simulating a strong activity at the end of the potential core region of the jet at $x / D=5$ (see Fig. 25, left). The fluctuating part of the resulting pressure-time signal is dominated by truncation effects (see Fig. 25, right). This truncation effect can be 

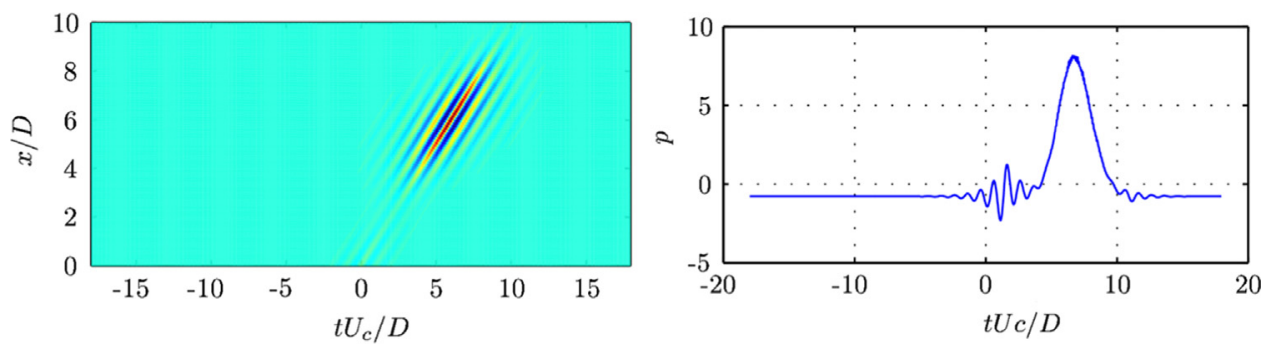

Fig. 25. Left: Resulting space-time distribution of the source calculated using a distribution correlated monopoles convecting with constant speed along a jet-axis. Right: Far-field pressure fluctuations calculated using a free-space Green's function.
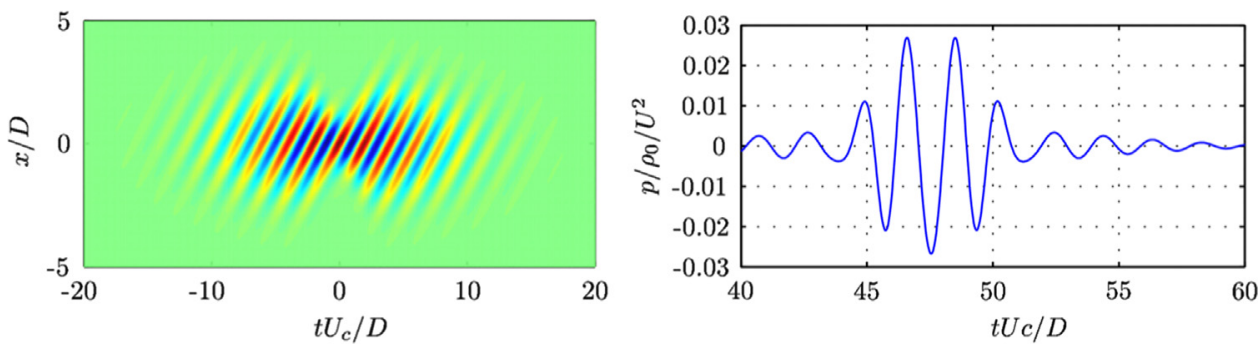

Fig. 26. Left: Space-time distribution of sources modeled by jittering wave-packets with varying amplitude and spatial extends. Right: Resulting far-field pressure fluctuations.

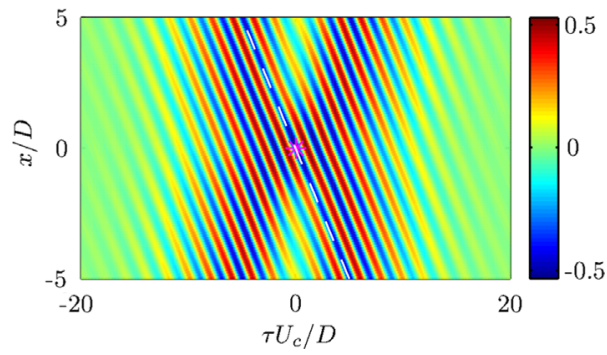

Fig. 27. Resulting space-time distribution of the correlation coefficient between the source and the far-field pressure. The magenta cross marks the position of the maximum value. The dashed line depicts the time delay based on the convection velocity. (For interpretation of the references to color in this figure caption, the reader is referred to the web version of this paper.)

avoided by using a numerical model based on jittering wave-packets with varying amplitude and spatial extents [10] (see Fig. 26, left). Here the resulting pressure signal is clearly dominated by the intermittency of the source signal, showing high amplitude burst (see Fig. 26, right). In case of the jittering wave-packets model the correlation between the source signal and the far-field is maximal for time delays, corresponding to the sound travel time from the source to the observer (see Fig. 27). The results verify the approach documented in the literature of analyzing the spatial and temporal evolution of the coefficient matrix in order to identify flow regions which are subject to the same physical phenomenon as the aeroacoustic source [33].

Written by Arne Henning (arne.henning@dlr.de) DLR, Germany.

\subsection{Acoustic-vortical wave equations in terms of total enthalpy as generalized acoustic variable}

The effects of vortical mean flows on the propagation of acoustic waves are numerous, from simple convection effects to instabilities in the acoustic phenomena, including absorption, reflection and refraction effects. Therefore, the role of vorticity in acoustic propagation besides noise generation has been a subject of controversial discussions since the foundation of aeroacoustics [46]. A theoretical investigation leads to the derivation of a family of scalar operators for aeroacoustics based in total enthalpy terms and including mean vorticity effects in the propagation. The selection of such a variable (total enthalpy) has been justified in [41,15]. Three models have been proposed in [41] (i) the high vorticity effects model (HVE); (ii) the medium vorticity effects model (MVE); and (iii) a model derived as an asymptotic expansion from Goldstein's analogy [27], Goldstein-Perez asymptotic model (GPA). All the previous models have been compared with a LEE solution and the classic convected wave equation POT) obtaining promising results. The cases selected for the comparisons were: (i) 
(a)
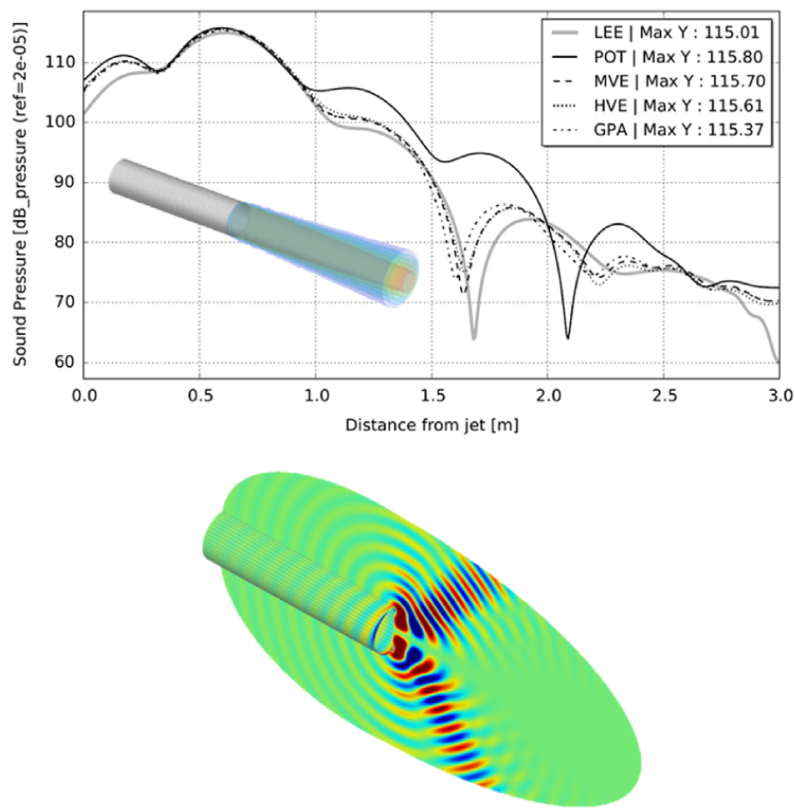

(b)
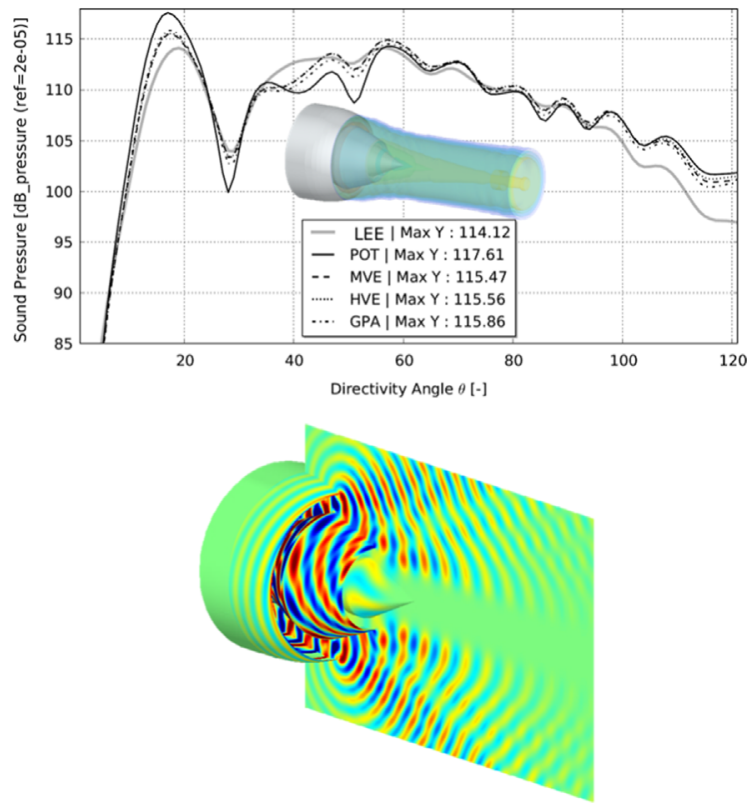

Fig. 28. (a) Cylindrical jet flow $(f=1500 \mathrm{~Hz}, n=1, m=2$, VJET $=150 \mathrm{~m} / \mathrm{s}$ ). Directivity (top) and pressure map (bottom) are presented(HVE model). (b) Realistic aircraft engine $(f=1000 \mathrm{~Hz}, n=1, m=2)$. Directivity (top) and pressure map (bottom) are presented (HVE model).

acoustic propagation of duct modes in a cylindrical jet configurations (see Fig. 28a); (ii) acoustic propagation in a realistic aircraft engine configuration (see Fig. 28b).

Written by Cesar Legendre, Free-Field Technologies, Belgium (cesar.legendre@fft.be).

\subsection{Improving the performance of pressure fluctuation measurements beneath a turbulent boundary layer in a wake flow}

A consortium of German automotive manufacturers investigated the noise generated by the air flow around the A-pillar and the side mirror of a generic vehicle model and the noise transmission through the front side window. The DLR was responsible for the optimization of the microphone distribution on the side window and the post processing to distinguish between acoustic and hydrodynamic pressure fluctuations [29,32].

The distribution of the microphones within the side window was optimized to increase ratio of the main lobe to side lobes with the limited number of microphones and space at hand.

A loudspeaker was placed in the side mirror in order to determine the array performance. Wavenumber space was chosen for display (Fig. 29) as it reveals different characteristics of the pressure fluctuations: acoustic components of the pressure propagate at the speed of sound. They appear inside the acoustic Mach-ellipse. Pressure fluctuations outside this ellipse are regarded as hydrodynamic fluctuations. Their energy is mainly concentrated in the convective ridge. Its position represents the convective flow speed [30].

For a consistent display of the results a slice through the $(k x, k y)$-plane at $k y=0$ was extracted for each frequency and combined in a $(k x, f)$ diagram (Fig. 30a). The acoustic part is clearly visible extending at a steep angle from the origin at $(k x=0, f=0)$. The convective ridge is very distinct as well having a moderate inclination. The threshold at which the acoustic signal from the loudspeaker could be retrieved in the measurement was considerably reduced to a frequency of $600 \mathrm{~Hz}$.

Written by Carsten Spehr (Carsten.Spehr@dlr.de), Stefan Haxter, DLR, Germany.

\subsection{Improved time domain impedance formulation for confined flows with non-uniform mean flow}

The utilization of sound-absorbing materials to control emitted noise level is of crucial importance for aeroacoustic applications. In this framework, numerical prediction techniques can be used analyze the effectiveness and physical behavior of different materials and liner geometrical configurations already in a design phase. Due to the known instability of the Ingard-Myers condition [59], an impedance boundary condition is complex to integrate in aeroacoustic time-domain solvers, which offer a clear advantage over frequency-domain methods in the resolution of large scale problems, transient wave simulations and nonlinear investigations. KU Leuven developed in the past [57] and recently improved a time domain impedance boundary condition based on recursive convolution method [2]. The new 

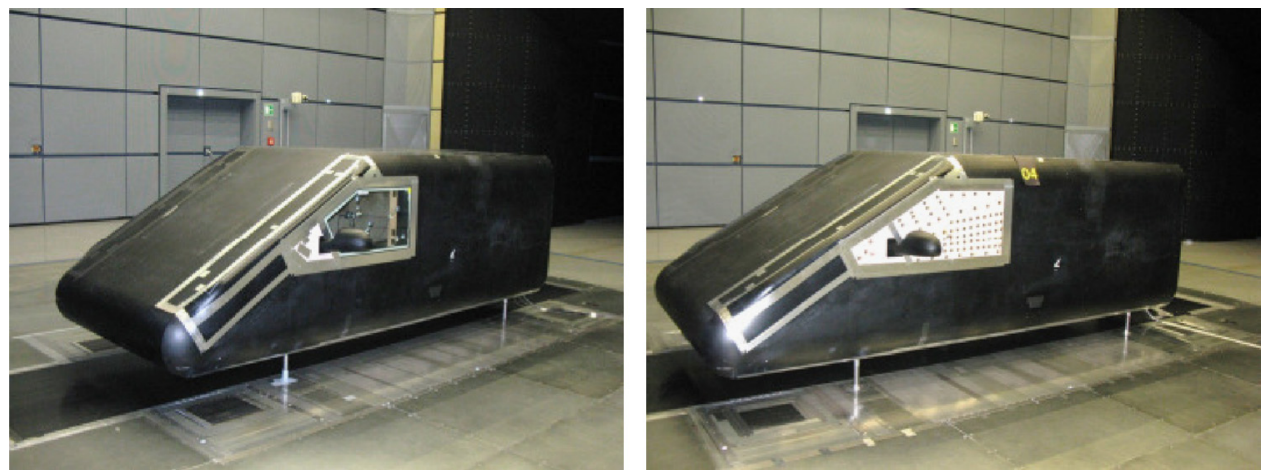

Fig. 29. Wind-tunnel test: glass module (left), sensor module (right) [29].

(a)

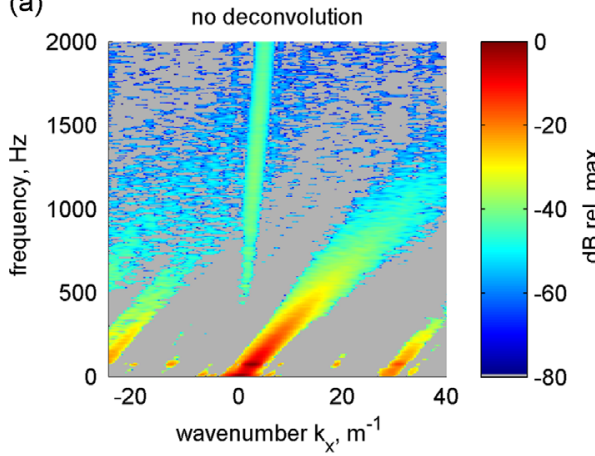

(b)

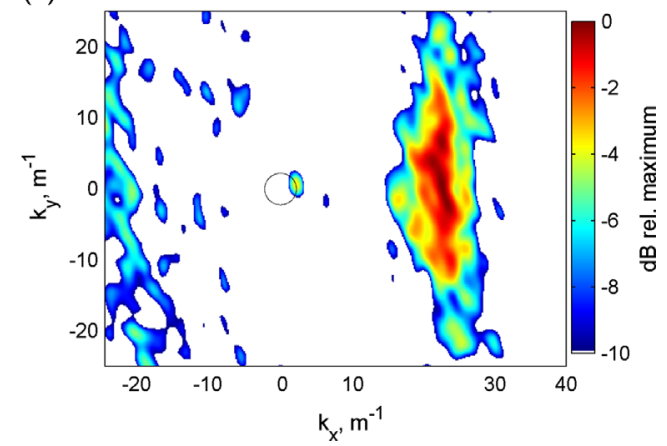

Fig. 30. (a) Wavenumber spectrum at $f=751 \mathrm{~Hz}$ at a flow speed of $140 \mathrm{~km} / \mathrm{h}$; The convective ridge is visible on the right. An acoustic source is identified as well, lying on the edge of the acoustic ellipse in the center. (b) Wavenumber-frequency-diagram at $k y=0$ : Different inclinations of the pressure fluctuations represent different propagation mechanisms. The loudspeaker signal can be identified as low as $600 \mathrm{~Hz}$.

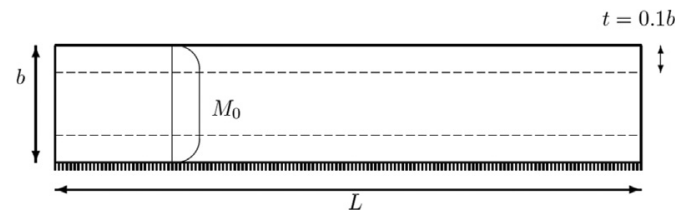

Fig. 31. Test case configuration.

formulation, implemented in an in house time-domain Runge-Kutta discontinuous Galerkin solver for LEE (Linearized Euler Equations) and LNSE (Linearised Navier-Stokes Equations), account for non-uniform substages within a time step in an optimized RK time-marching scheme [64].

Assuming the pressure to be piecewise linear within a time step and approximating the impedance model with a number of first- and second-order systems, the convolution integral is discretized and solved using some constant terms over the substages and some accumulators which reduce the computational cost to solve the convolution integral. The boundary condition can properly describe the interaction between a soft wall and a non-uniform mean flow with finite thickness boundary layer, thus avoiding the use of the Ingard-Myers condition. The boundary condition is verified for the case of a non-uniform mean flow in a 2D rectangular duct, Fig. 31, comparing the wall impedance imposed in the model and the one retrieved after the simulation. Fig. 32 shows a relative error smaller than 3.5

Written by Antonio Ammirati (antonio.ammirati@kuleuven.be), Herve Denayer, Wim De Roeck, Wim Desmet, KU Leuven, Belgium.

\subsection{Experimental aerodynamic and aeroacoustic characterization of a butterfly valve with inflow distortion}

Environmental Control Systems (ECS) are key contributors to the acoustic nuissance within the aircraft cabin and around a grounded aircraft. Therefore the European IDEALVENT project aims at gaining further insight in the noise generation and radiation mechanisms in ECS systems with specific focus on the influence of multi-component interactions on the aeroacoustic behavior. At KU Leuven, a measurement campaign has been carried out on a simplified ECS mockup, consisting 


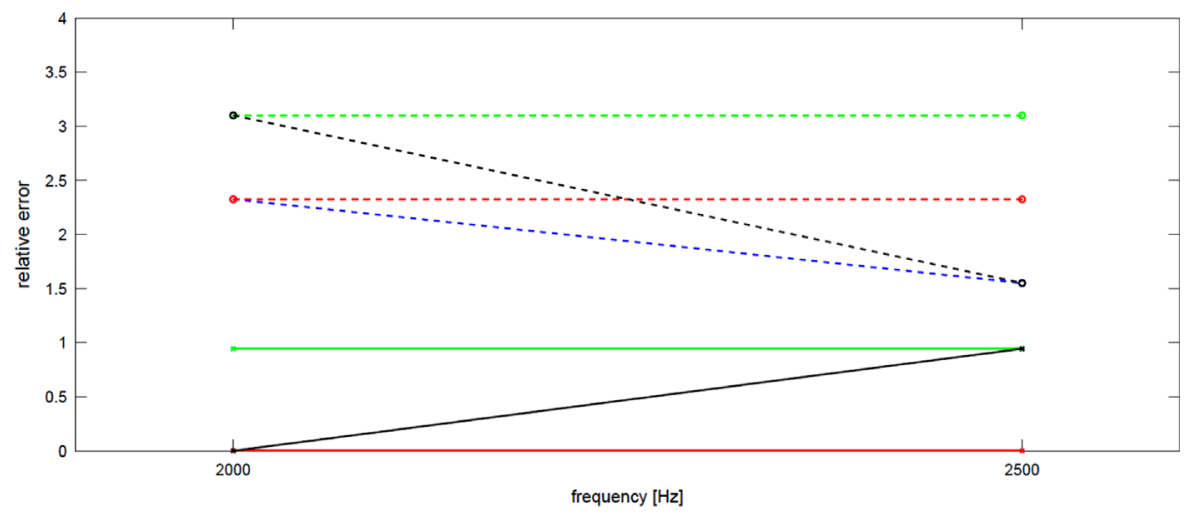

Fig. 32. Relative error between wall impedance retrieved and wall impedance imposed as a function of frequency for non-uniform mean flow (full lines real part of the error, dashed lines imaginary part of the error, blue lines $\mathrm{M} 0=0.11$, red lines $\mathrm{M} 0=0.04$, green. (For interpretation of the references to color in this figure caption, the reader is referred to the web version of this paper.)

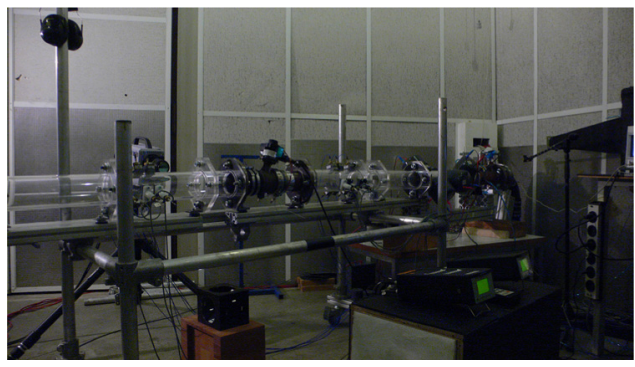

Fig. 33. Test rig layout.

of a butterfly valve with and without upstream $90^{\circ}$ bend (Fig. 33). Hot wire anemometry and particle image velocimetry measurements [65] provide aerodynamic inlet conditions and validation data for future numerical simulations of the mockup. The acoustic characterization is based on a passive and active multiport formulation. This is an extension of the classical two-ports formulation $[40,61]$ to higher frequencies, accounting for the presence of a limited set of higher-order duct modes. The passive multiport approach was first validated on the benchmark case of an empty duct (Fig. 34), showing an excellent comparison with the expected analytical solution. The same methodology was afterwards applied to the ECS valve and valve with bend mockups, to characterize the installation effects caused by the inflow distortion. The noise generation of both mockups was characterized in a second step, by carrying out an active multiport characterization (Fig. 35).

Written by Herve Denayer(Herve.Denayer@kuleuven.be), Wim De Roeck, Wim Desmet, KU Leuven, Belgium.

\section{Miscellaneous topics}

\subsection{Nonlinear asymptotic impedance model for a Helmholtz resonator liner}

In order to study acoustic liners made of an array of Helmholtz resonator cells, an asymptotically systematic 2nd-order solution of the nonlinear Helmholtz resonator equation has been constructed, based on a small parameter derived from the excitation amplitude. Analytical expressions were obtained for the liner impedance, representing nonlinear behaviour due to vortex shedding close to resonance, and mainly linear behaviour away from resonance.

The real part of the found impedance (the resistance) shows the usual characteristic behaviour as a function of frequency, namely a maximum at or near the resonance frequency and a decay along both sides. All values increase with the amplitude, but slightly more for the frequencies less than resonance. The imaginary part of the impedance (the reactance) is linear in frequency in a way that it vanishes at resonance and is practically independent of the amplitude.

To leading order, the usually assumed form of the liners's resistance, $a+b|v|$, is recovered, but the asymptotic analysis allows higher order corrections, which indeed are shown to be important and relevant for practical configurations involving high amplitudes, as may be seen from Fig. 36, displaying as a function of frequency the real and imaginary parts with a 0th or a 0 th +1 st order corrections in a realistic configuration.

Written by Deepesh K. Singh (d.k.singh@tue.nl) Sjoerd W. Rienstra (s.w.rienstra@tue.nl) Technische Universiteit Eindhoven, The Netherlands. 

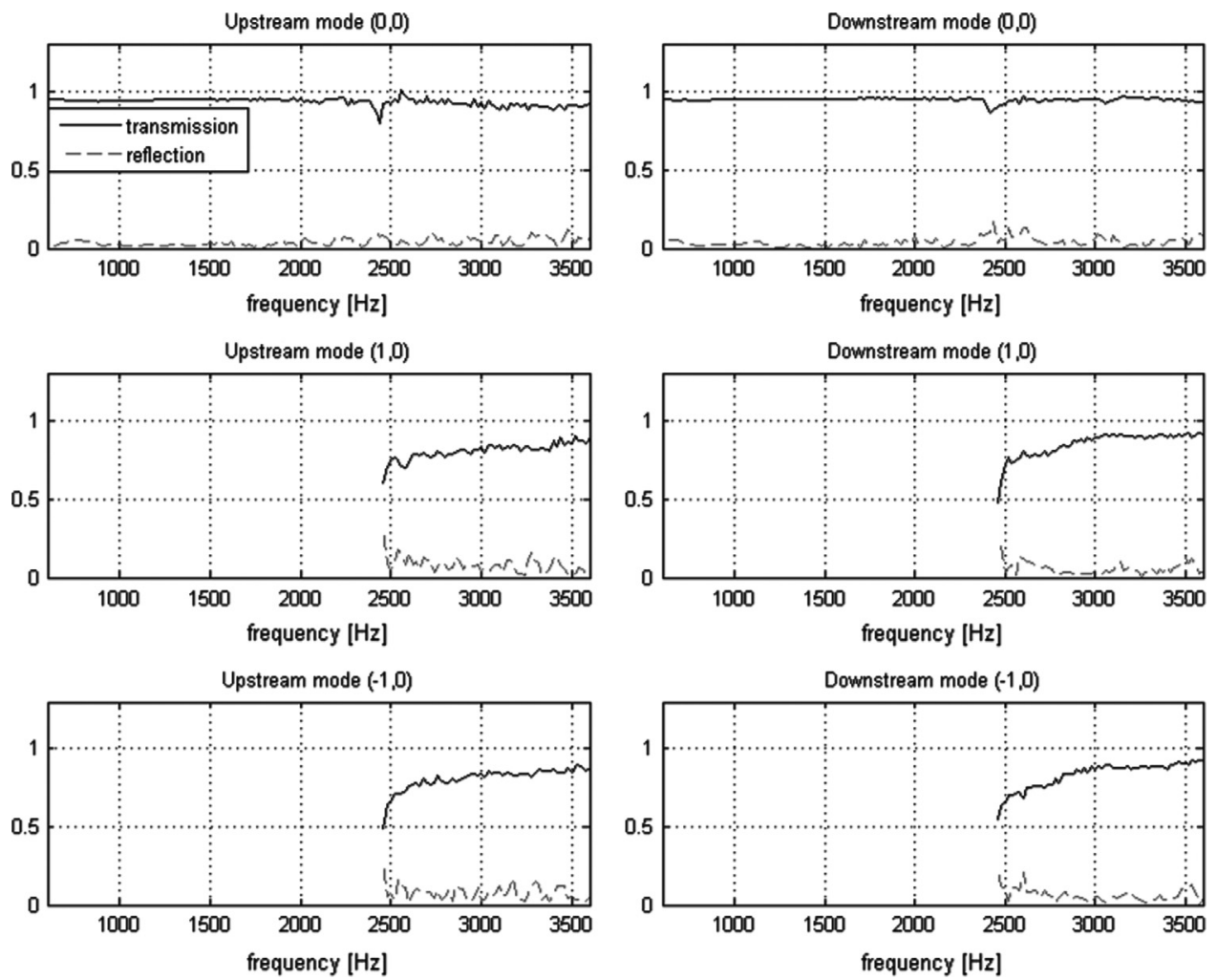

Fig. 34. Reflection and transmission scatter matrix coefficients of an empty for the, from top to bottom, (0, 0), (1, 0) and ( $-1,0)$-mode (left: upstream modes, right: downstream modes).
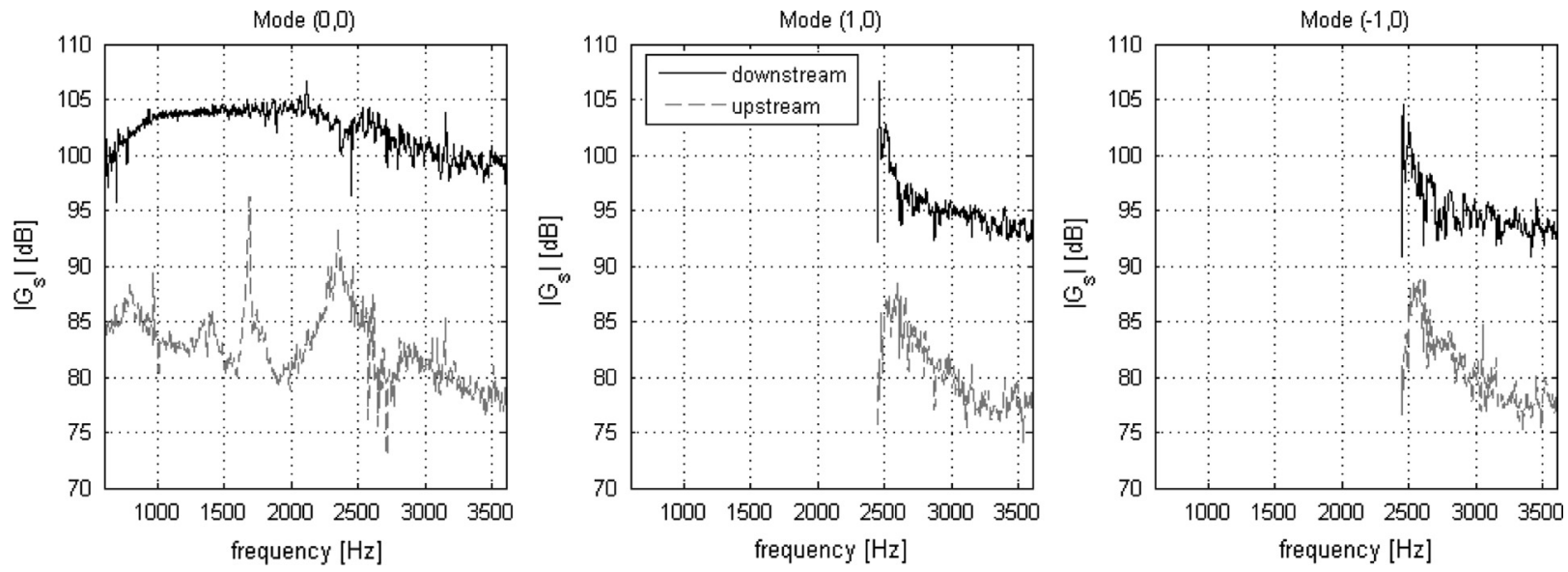

Fig. 35. Magnitude $(\mathrm{dB})$ of the active source vector coefficients for the valve measurements for the, from left to right, $(0,0)$ mode, $(1,0)$ mode and $(-1$, 0) mode.

\subsection{Effects of aircraft engine installation on ground sound pressure levels}

Reducing the noise levels around airports remains a key issue due to traffic increase, even if aircraft is more and more silent. However, it seems that a new gap cannot be expected keeping the present technology and several novel airplane concepts are considered for the next decades [58,24]. The shielding of turbofan noise with fuselage, wings or stabilizer could be beneficial for sound pressure radiated towards the ground [56]. Present work focuses on comparisons between engine under or over the wing, based on parametric studies avoiding long CFD-CAA calculations. Three fast complementary methods have been implemented to evaluate the effect of engine installation on sound pressure levels on the ground: an analytical model of reflection and diffraction [9], a boundary element model (BEM), and tests on a 1/10-scale wing model in a large anechoic chamber. Each method applies to a preferred frequency range (high, low, and medium, respectively), but 

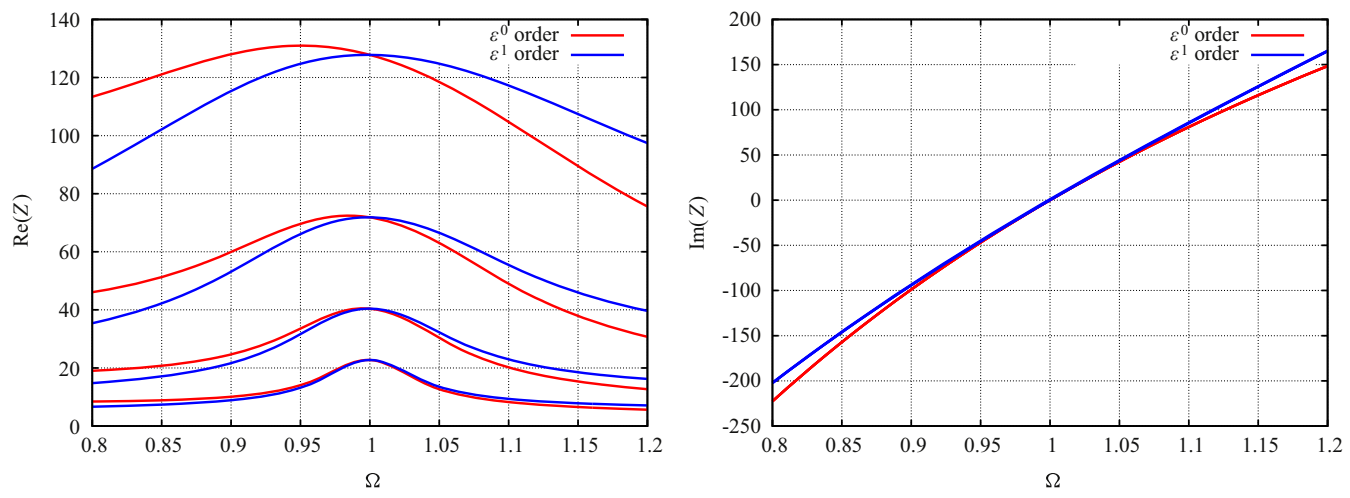

Fig. 36. 0 -th and 0 -th +1 -st order approx. impedance in realistic configuration for $100 \mathrm{~dB}, 110 \mathrm{~dB}, 120 \mathrm{~dB}$ and $130 \mathrm{~dB}$.

(a)

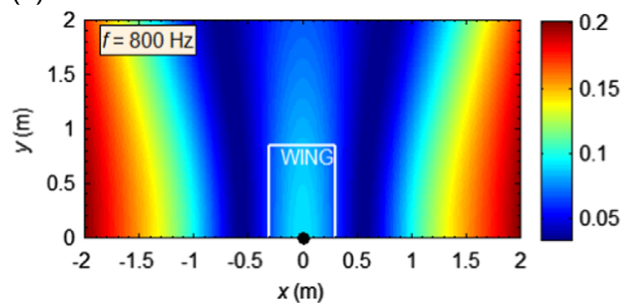

(b)

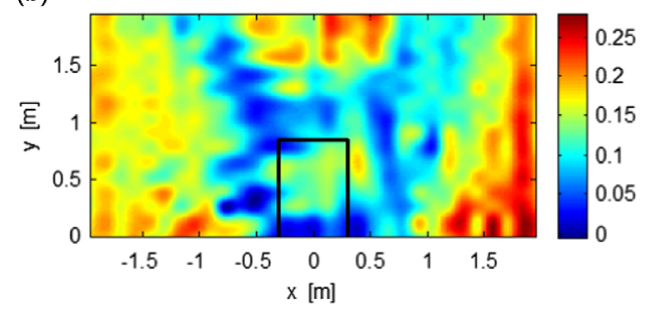

Fig. 37. RMS value of sound pressure (in Pa) on a horizontal surface, $1.60 \mathrm{~m}$ below the wing (at scale $1 / 10$ ). Source in $x=0, y=0$, at $30 \mathrm{~cm}$ over the wing, frequency 800 Hz. (a) Analytical map. (b) Experimental result.

(a)

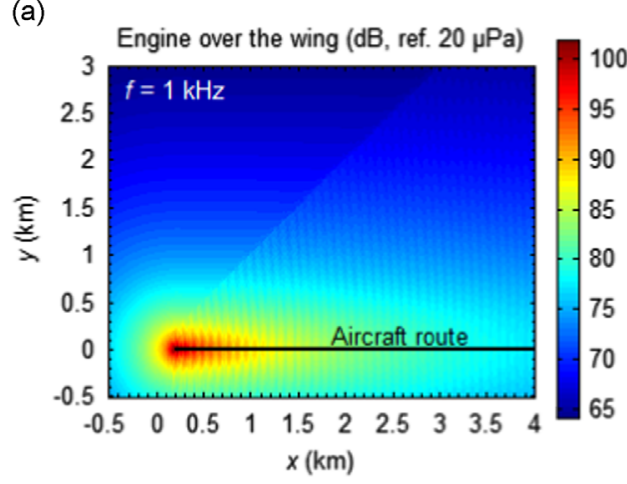

(b)

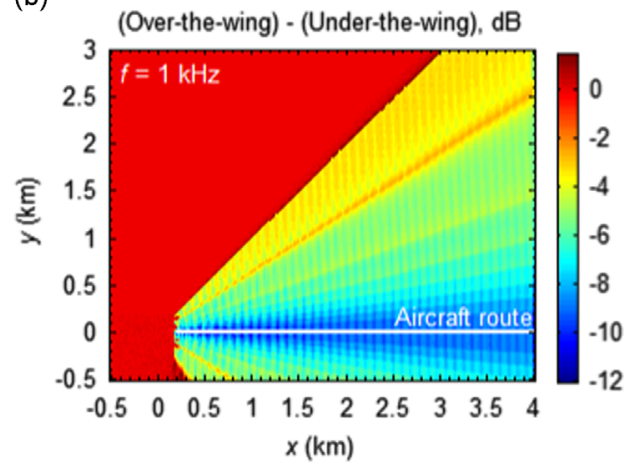

Fig. 38. (a) Maximum sound pressure level, SPL, on the ground during takeoff due to a point source simulating an engine. Source over the wing. (b) SPL (over-the-wing) and SPL (under-the-wing).

some appraisals can be cross-checked (Fig. 37a). Results simulating a takeoff flight show that a judicious location of the engines can be fruitful in terms of noise management. An engine over the wing can provide a gain of 4-10 dB in the shadow zone on several kilometers around the aircraft route where a conventional design is the noisiest (Fig. 38a). Present study should be extended in the future to take into account the non-compactness of the acoustic sources and the refraction due to the flow. Other constraints in an aircraft design should also be examined (aerodynamic performance, structure resistance, fuel consumption).

Written by Serge Lewy (Serge.Lewy@onera.fr), ONERA, France, Nicolas Joly, Jean-Claude Pascal, ENSIM - LAUM, UMR CNRS 6613, University of Maine, Le Mans, France.

\subsection{Sound attenuation in hot and pressurized flow}

It is common practice in duct acoustics to account for viscothermal losses at the walls only and neglect dissipative effects within the bulk of the fluid. Recent experiments [38] revealed an increasing attenuation at elevated pressure and temperature, which cannot be justified by the viscothermal losses at the walls alone. It was confirmed by theory and experiment [39] that the increased absorption is attributed to the molecular relaxation losses within the fluid. As a 
(a)

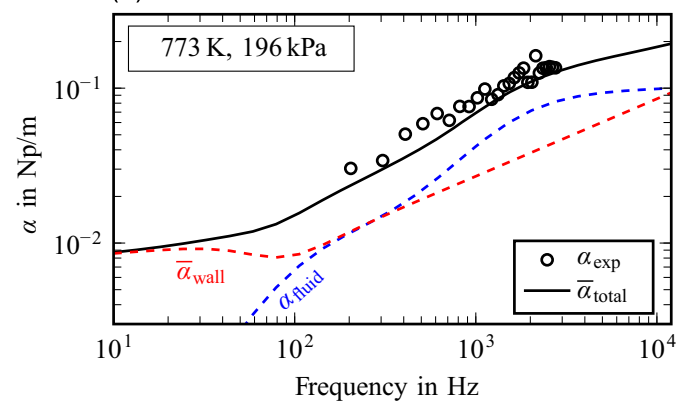

(b)

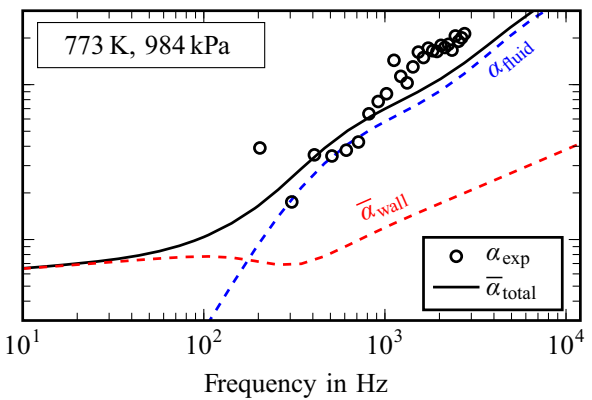

Fig. 39. Comparison of the attenuation coefficient determined experimentally and theoretical predictions of the total losses, including the losses at the wall and the losses within the fluid at (a) $773 \mathrm{~K}, 196 \mathrm{kPa}$ and (b) $773 \mathrm{~K}, 984 \mathrm{kPa}$

conclusion, a formulation of the total losses, combining the wall losses (viscothermal and turbulent boundary-layer interaction) and the losses within the fluid (viscothermal and molecular relaxation), was presented in [39]. The total losses should be used when modeling the sound attenuation in hot and pressurized flow, while the wall losses alone are sufficient at normal atmospheric conditions. The theoretical predictions were confirmed by measurements in the Hot Acoustic Test Rig (HAT) at various temperature and pressure conditions (see two examples in Fig. 39). Our experience with the Hot Acoustic Test Rig has shown that the quality of the results improves significantly when taking the total losses into account during the analysis, for example when applying a wave decomposition to liner or indirect combustion noise measurements. Generally, the findings are of great importance when studying the sound propagation in the hot gas path of aero-engines.

Written by C. Lahiri (claus.lahiri@dlr.de), K. Knobloch, F. Bake, and L. Enghardt, DLR, Germany.

\section{References}

[1] R. Akkermans, M. Pott-Pollenske, H. Buchholz, J. Delfs, D. Almonait. Installation effects of a propeller mounted on a high-lift wing with a coanda flap. Part I. Aeroacoustic experiments. Proceedings of the 20th AIAA/CEAS Aeroacoustics Conference, AIAA (2014) $2014-3191$.

[2] A. Ammirati, H. Denayer, W. De Roeck, W. Desmet. Validation of a time domain impedance formulation for the case of a $2 \mathrm{~d}$ rectangular lined duct with a non-uniform mean-flow with a finite boundary layer thickness, Proceedings of ISMA2014, 15-17 September 2014, p. 20.

[3] H. Atassi, A. Ali, O. Atassi, I. Vinogradov, Scattering of incident disturbances by an annular cascade in a swirling flow, Journal of Fluid Mechanics 499 (2004) 111-138.

[4] M. Bauer, J. Dierke, R. Ewert, Application of a discontinuous Galerkin method to discretize acoustic perturbation equations, AIAA Journal 49 (5) (2011) 898-908.

[5] C. Beckmann, J. Hohe, Effects of material uncertainty in the structural response of metal foam core sandwich beams, Composite Structures 113 (0) (2014) 382-395.

[6] G. Bernardini, J. Serafini, S. Ianniello, M. Gennaretti, Assessment of computational models for the effect of aeroelasticity on bvi noise prediction, International Journal of Aeroacoustics 6 (3) (2007) 199-222.

[7] G. Bernardini, C. Testa, M. Gennaretti. Tiltrotor cabin noise control through smart actuators, Journal of Vibration and Control, http://dx.doi.org/10.1177/ 1077546311421516 , in press.

[8] D.E. Breakey, J.A. Fitzpatrick, C. Meskell. Aeroacoustic source analysis using time-resolved piv in a free jet, Experiments in Fluids 54 (5) (2013), http://dx. doi.org/10.1007/s00348-013-1531-5.

[9] E. Broadbent, Noise shielding for aircraft, Progress in Aerospace Sciences 17 (0) (1976) 231-268.

[10] A.V. Cavalieri, P. Jordan, A. Agarwal, Y. Gervais, Jittering wave-packet models for subsonic jet noise, Journal of Sound and Vibration 330 (1819) (2011) 4474-4492.

[11] V. Clair, C. Polacsek, T. Le Garrec, M. Jacob. Numerical predictions of turbulence-cascade interaction noise using caa with a stochastic model. in: Proceedings of the 20th AIAA/CEAS Aeroacoustics Conference, AIAA (2014) 2014-2453.

[12] L. De Santana, C. Schram, W. Desmet, 2014. A boundary element method iterative procedure to compute the compressible response of an airfoil subjected to incoming turbulence, Proceedings of the 20th AIAA/CEAS Aeroacoustics Conference, Atlanta.

[13] J. Delfs, B. Faßmann, N. Lippitz, M. Mößner, L. Müller, K. Rurkowska, S. Uphoff, Sfb 880: aeroacoustic research for low noise take-off and landing, CEAS Aeronautical Journal 5 (4) (2014) 403-417.

[14] J. Dierke, R. Akkermans, J. Delfs, R. Ewert, Installation effects of a propeller mounted on a high-lift wing with a coanda flap. Part II. Numerical investigation and experimental validation, Proceedings of the 20th AIAA/CEAS Aeroacoustics Conference, AIAA (2014) $2014-3189$.

[15] P. Doak, Fluctuating total enthalpy as the basic generalized acoustic field, Theoretical and Computational Fluid Dynamics 10 (1-4) (1998) 115-133.

[16] V. D'Alessandro, G. Petrone, S.D. Rosa, F. Franco, Modelling of aluminium foam sandwich panels, Smart Structures and Systems 13 (2) (2014) 615-636.

[17] I. Duran, A.S. Morgans, On the reflection and transmission of circumferential waves through nozzles, Journal of Fluid Mechanics (2015) http://dx.doi. org/10.1017/jfm.2015.247, in press.

[18] B. Efimtsov, Characteristics of the field of turbulent wall pressure fluctuations at large Reynolds numbers, Soviet Physics Acoustics (1982) 28.

[19] E. Envia, J. Coupland, Fan broadband noise prediction workshop, Proceedings of the 21th AIAA/CEAS Aeroacoustics Conference, 2015.

[20] R. Ewert, Slat noise trend predictions using caa with stochastic sources from a random particle mesh method (rpm), Proceedings of the 12th AIAA/CEAS Aeroacoustics Conference, AIAA 2006-2667.

[21] R. Ewert, J. Dierke, A. Neifeld, S. Moghadam, Linear and non-linear perturbation equations with relaxation source terms for forced eddy simulation of aeroacoustic sound generation, Proceedings of the 20th AIAA/CEAS Aeroacoustics Conference, AIAA 2014-3053.

[22] M. Gennaretti, G. Bernardini, Novel boundary integral formulation for blade-vortex interaction aerodynamics of helicopter rotors, AIAA Journal 45 (6) (2007) 1169-1176.

[23] M. Gennaretti, M.M. Colella, G. Bernardini, Prediction of tiltrotor vibratory loads with inclusion of wing-proprotor aerodynamic interaction, Journal of Aircraft 47 (2010) 71-79.

[24] C.H. Gerhold, L.R. Clark, M.H. Dunn, J. Tweed, Investigation of acoustical shielding by a wedge-shaped airframe, Journal of Sound and Vibration 294 (12) (2006) 49-63. 
[25] C.S. Goh, A.S. Morgans, Phase prediction of the response of choked nozzle to entropy and acoustic disturbances, Journal of Sound and Vibration 330 (2011), 5184-5198.

[26] C.S. Goh, A.S. Morgans, The influence of entropy waves on the thermoacoustic stability of a model combustor, Combustion Science and Technology 185 (2013) 249-268.

[27] M.E. Goldstein, Unsteady vortical and entropic distortions of potential flows round arbitrary obstacles, Journal of Fluid Mechanics 89 (12) (1978) 433-468.

[28] G. Grasso, J. Christophe, C. Schram, T. Verstraete, Influence of the noise prediction model on the aeroacoustic optimization of a contra-rotating fan, Proceedings of the 20th AIAA/CEAS Aeroacoustics Conference, Atlanta, 2014, No. AIAA-2014-2611.

[29] M. Hartmann, J. Ocker, T. Lemke, A. Mutzke, V. Schwarz, H. Tokuno, R. Toppinga, P. Unterlechner, G. Wickern, Wind noise caused by the side-mirror and a-pillar of a generic vehicle model, Proceedings of the 18th AIAA/CEAS Aeroacoustics Conference, AIAA $2012-2205$.

[30] S. Haxter, C. Spehr, Two-dimensional evaluation of turbulent boundary layer pressure fluctuations at cruise flight conditions, Proceedings of the 18th AIAA/CEAS Aeroacoustics Conference, 2012.

[31] S. Haxter, C. Spehr, Listening to turbulence: Measuring coherence decay at different positions on an aircraft in cruise flight, Proceedings of the 20th AIAA/CEAS Aeroacoustics Conference, AIAA 2014-3064.

[32] S. Haxter, C. Spehr, M. Hartmann, J. Ocker, H. Tokuno, , G. Wickern, Improving the performance of aeroacoustic measurements beneath a turbulent boundary layer in a wake flow, Proceedings of the 20th AIAA/CEAS Aeroacoustics Conference, 2014.

[33] A. Henning, K. Kaepernick, K. Ehrenfried, L. Koop, A. Dillmann, Investigation of aeroacoustic noise generation by simultaneous particle image velocimetry and microphone measurements, Experiments in Fluids 45 (6) (2008) 1073-1085.

[34] A. Henning, B. Wrede, A. Schröder, About the ambiguity of noise source localization based on the causality correlation technique, Proceedings of 17th Internatinal Symposium on Application Laser Techniques to Fluid Mechanics, July 2014.

[35] M. Herr, M. Pott-Pollenske, R. Ewert, D. Boenke, J. Siebert, J. Delfs, A. Rudenko, A. Büscher, H. Friedel, I. Mariotti, Large-scale studies on slat noise reduction, Proceedings of the 21th AIAA/CEAS Aeroacoustics Conference, 2015.

[36] M. Herr, K.-S. Rossignol, J. Delfs, M. Mößner, N. Lippitz, Specifcation of porous materials for low-noise trailing-edge applications, Proceedings of the 20th AIAA/CEAS Aeroacoustics Conference, 2014.

[37] F. Kameier, W. Neise, Rotating blade flow instability as a source of nose in axial turbomachines, Journal of Sound and Vibration 203 (5) (1997) 833-853.

[38] C. Lahiri, K. Knobloch, F. Bake, L. Enghardt, Acoustic measurements of perforated liners in hot and pressurized flow, in: ASME Tubo Expo 2013, San Antonio, Texas, 3-7 June 2013, 2013-94674.

[39] C. Lahiri, K. Knobloch, F. Bake, L. Enghardt, Attenuation of sound in wide ducts with flow at elevated pressure and temperature, Journal of Sound and Vibration 333 (2014) 3440-3458.

[40] J. Lavrentjev, M. Abom, Characterization of fluid machines as acoustic multi-port sources, Journal of Sound and Vibration 197 (1996) 1-16.

[41] C. Legendre, On the Interactions of Sound Waves and Vortices, Ph.D. Thesis, Universit Libre de Bruxelles, 2015.

[42] T. Lu, A. Hess, M. Ashby, Sound absorption in metallic foams, Journal of Applied Physics 85 (11) (1999) $7528-7539$.

[43] A. McAlpine, M. Fisher, On the prediction of "buzz-saw" noise in aero-engine inlet ducts, Journal of Fluid Mechanics 248 (2001) $123-149$.

[44] S. Modini, G. Graziani, G. Bernardini, M. Gennaretti, Parallel blade-vortex interaction modelling for helicopter rotor noise control synthesis, International Journal of Aeroacoustics 13 (7-8) (2014) 587-606.

[45] A.S. Morgans, C.S. Goh, J.A. Dahan, The dissipation and shear dispersion of entropy waves in combustor thermoacoustics, Journal of Fluid Mechanics (2013) 733.

[46] P. Morris, Jet noise prediction: past, present and future, Canadian Acoustics 35 (3) (2007).

[47] A. Neifeld, R. Ewert, D. Keller, M. Steger, Towards prediction of jet noise installation effect using stochastic source modeling, Proceedings of the 20th AIAA/CEAS Aeroacoustics Conference, AIAA 2014-3059.

[48] D. Palumbo, The variance of convection velocity in the turbulent boundary layer and its effect on coherence length, Journal of Sound and Vibration 332 (2013) 3692-3705.

[49] B. Pardowitz, U. Tapken, L. Enghardt, Time-resolved rotating instability waves in an annular cascade, Proceedings of the 18th AIAA/CEAS Aeroacoustics Conference, AIAA 2012-2132.

[50] B. Pardowitz, U. Tapken, L. Neuhaus, L. Enghardt, Experiments on an axial fan stage: time-resolved analysis of rotating instability modes, Journal of Engineering for Gas Turbines and Power 137 (2014) 6.

[51] B. Pardowitz, U. Tapken, R. Sorge, P.-U. Thamsen, L. Enghardt, Rotating instability in an annular cascade: detailed analysis of the instationary flow phenomena, ASME Journal of Turbomachinery 136 (2013) 6.

[52] G. Pickett, Prediction on the spectral content of combination tone noise, Journal of Aircraft 9 (9) (1972) 658-663.

[53] H. Posson, M. Roger, Experimental validation of a cascade response function for fan broadband noise predictions, AIAA Journal 49 (9) (2011) 1907-1918.

[54] M. Pott-Pollenske, J. Delfs, J. Reichenberger, A testbed for large scale and high Reynolds number airframe noise research, Proceedings of the 19th AIAA/ CEAS Aeroacoustics Conference, AIAA 2013-2260.

[55] M. Pott-Pollenske, H. Friedel, Aeroacoustic characteristics of externally blown flap systems, Proceedings of the 17th AIAA/CEAS Aeroacoustics Conference, AIAA 2011-2729.

[56] S. Powell, A. Sóbester, P. Joseph, Fan broadband noise shielding for over-wing engines, Journal of Sound and Vibration 331 (23) (2012) 5054-5068.

[57] Y. Reymen, M. Baelmans, W. Desmet, Efficient implemantation of tam and auriault's time-domain impedance boundary condition, AIAA Journal 46 (9) (2008) 2368-2376.

[58] J. Ricouard, R. Davy, P. Loheac, A. Moore, O. Piccin, Rosas wind tunnel test campaign dedicated to unconventional aircraft concepts study, Proceedings of the 10th AIAA/CEAS Aeroacoustics Conference, Manchester, AIAA 2004-2867.

[59] S. Rienstra, Impedance models in time domain, including the extended Helmholtz resonator model, Proceedings of the 12th AIAA/CEAS Aeroacoustics Conference, AIAA 2006-2686.

[60] M. Roger, S. Moreau, Back-scattering correction and further extensions of Amiet's trailing-edge noise model. Part I. Theory, Journal of Sound and Vibration 286 (3) (2005) 477-506.

[61] S. Sack, M. Abom, C. Schram, K. Kucukcoskun, Generation and scattering of acoustic modes in ducts with flow, Proceedings of the 20th AIAA/CEAS Aeroacoustics Conference, Atlanta, 2014, No. AIAA-2014-3115.

[62] C.K.W. Tam, L. Auriault, Jet mixing noise from fine-scale turbulence, AIAA Journal 37 (2) (1999) 145-153.

[63] J. Thisse, C. Polacsek, S. Lewy, A. Lafitte, On the generation and propagation of multiple pure tones inside turbofans at transonic regime, Proceedings of the 20th AIAA/CEAS Aeroacoustics Conference, AIAA 2014-3104.

[64] T. Toulorge, W. Desmet, Optimal Runge-Kutta schemes for discontinuous Galerkin space discretizations applied to wave propagation problems, Journal of Computational Physics 231 (4) (2012) 2067-2091.

[65] M. Vanierschot, H. Denayer, W.D. Roeck, W. Desmet, Experimental study of the valve-bend interaction in an aircraft ecs-system, 11th International Conference on Heat Transfer, Fluid Mechanics and Thermodynamics, Kruger National Park, South Africa, 2015.

[66] X. Wang, T.J. Lu, Optimized acoustic properties of cellular solids, The Journal of the Acoustical Society of America 106 (2) (1999) $756-765$.

[67] J. Yin, S. Ahmed, Helicopter main-rotor/tail-rotor interaction, Journal of the American helicopter society 45 (4) (2000) $293-302$.

[68] J. Yin, B.G. van der Wall, G.A. Wilke, Rotor aerodynamic and noise under influence of elastic blade motion and different fuselage modeling, in: 40th European Rotorcraft Forum (ERF), Southampton, UK, 2-5 September 2014. 